\title{
Gauss's Law Satisfying Energy-Conserving Semi-Implicit Particle-in-Cell Method
}

\author{
Yuxi Chen ${ }^{1 \mathrm{a}}$, Gábor Tóth ${ }^{\mathrm{a}}$ \\ ${ }^{a}$ Center for Space Environment Modeling, University of Michigan, Ann Arbor, Michigan \\ 48109, USA
}

\begin{abstract}
The Energy Conserving Semi-Implicit Method (ECSIM) introduced by Lapenta (2017) has many advantageous properties compared to the classical semiimplicit and explicit PIC methods. Most importantly, energy conservation eliminates the growth of the finite grid instability. We have implemented ECSIM in a different and more efficient manner than the original approach. More importantly, we have addressed two major shortcomings of the original ECSIM algorithm: there is no mechanism to enforce Gauss's law and there is no mechanism to reduce the numerical oscillations of the electric field. A classical approach to satisfy Gauss's law is to modify the electric field and its divergence using either an elliptic or a parabolic/hyperbolic correction based on the Generalized Lagrange Multiplier method. This correction, however, violates the energy conservation property, and the oscillations related to the finite grid instability reappear in the modified ECSIM scheme. We invented a new alternative approach: the particle positions are modified instead of the electric field in the correction step. Displacing the particles slightly does not change the energy conservation property, while it can satisfy Gauss's law by changing the charge density. We found that the new Gauss's Law satisfying Energy Conserving Semi-Implicit Method (GL-ECSIM) produces superior results compared to the original ECSIM algorithm. In some simulations, however, there are still some numerical oscillations present in the electric field. We attribute this to the simple finite difference discretization of the energy conserving implicit electric field solver. We modified the spatial discretization of the field solver to reduce these oscillations while only slightly violating the energy conservation properties. We demonstrate the improved
\end{abstract}

\footnotetext{
${ }^{1}$ Corresponding author. Email address: yuxichen@umich.edu
} 
quality of the GL-ECSIM method with several tests.

Keywords: Particle-in-cell (PIC). Semi-implicit particle-in-cell. energy conservation. Charge conservation. Gauss's law

\section{Introduction}

Conservation properties play an important role to avoid numerical instabilities for the particle-in-cell (PIC) method. The explicit PIC method, which is widely used due to its simplicity, conserves the total moment but tends to increase the total energy of the system by numerical heating. The implicit PIC method, which relaxes the temporal and spatial stability constraints, tends to decrease the system energy by numerical cooling. Fully implicit PIC schemes can achieve energy conservation by solving for the particle motions and electro-magnetic fields at the same time via a non-linear Newton-Krylov iterative solver [1, 2, 3, 4]. Recently, Lapenta [5] proposed an Energy Conserving Semi-Implicit Method (ECSIM) that conserves energy by ensuring the current used for electric field updating is the same as the current produced by moving particles.

Another important conservation law is related to Gauss's law:

$$
\nabla \cdot \mathbf{E}=4 \pi \rho
$$

where $\mathbf{E}$ is the electric field and $\rho$ is the electric charge density. Analytically, Gauss's law will be satisfied if the initial condition satisfies it and Ampère's law and the charge conservation equations are solved exactly. Ampère's law describes the evolution equation for the electric field:

$$
\frac{\partial \mathbf{E}}{\partial t}=c \nabla \times \mathbf{B}-4 \pi \mathbf{J}
$$

where $\mathbf{J}$ is the current density, $\mathbf{B}$ is the magnetic field vector and $c$ is the speed of light. The charge density evolves according to

$$
\frac{\partial \rho}{\partial t}+\nabla \cdot \mathbf{J}=0
$$

Taking the divergence of Ampère's law and using the charge conservation leads to

$$
\frac{\partial \nabla \cdot \mathbf{E}}{\partial t}=4 \pi \frac{\partial \rho}{\partial t}
$$


which means that Gauss's law is maintained as long as it holds initially.

The electromagnetic PIC methods usually update the electric field by solving Ampères law from the magnetic field and the current on a grid. This current is interpolated to the grid from the particles and does not necessarily satisfy the charge conservation equation. This discrepancy may accumulate and lead to significant violation of Gauss's law. Two classes of methods have been proposed to solve this numerical issue. One approach is enforcing the electric field to satisfy Gauss's law by applying a correction term to the electric field equation. The correction can be applied as an extra correction step, or added to the electric field solver directly. Boris' popular $\nabla \cdot \mathbf{E}$ error correction method [6, 7] solves a Poisson equation and reduces the error in Gauss's law to the iteration tolerance level. Marder [8] and Langdon [9] reduce the computational cost by replacing the Poisson solver with a local fix. Marder [8] calls the correction term as 'pseudo-current'. The idea of electric field correction is generalized by Assous et al. [10] and Munz et al. [11] in a generalized Lagrange multiplier (GLM) numerical framework, where new variables are introduced to the Maxwell's equations to constrain the errors in Gauss's law. The other class of methods does not require any electric field correction. Instead, these methods carefully design the algorithm so that the current assigned to the electric field solver satisfies the charge conservation equation and hence Gauss's law automatically . Buneman [12] developed the 'zero-order current weighting' algorithm, which uses an impulse current assignment when a particle crosses a cell boundary. Similarly, Morse and Nielson [13] proposed the 'first-order current weighting' method, where the current is assigned by area weighting and the particle motion is divided into two or three orthogonal moves. Villasenor and Buneman [14] introduced another area weighting method which does not require the orthogonal motion splitting. This scheme is generalized to any form-factor by Esirkepov [15]. Umeda et al. [16] developed an algorithm similar to Villasenor and Buneman [14 but assumes the particle trajectory is zigzag. Sokolov [17] introduced a method to conserve charge using an alternating order form-factor. Eastwood [18, 19] presented a general description of the charge conserving scheme for Cartesian and curvilinear grids. Besides these two classes of techniques, Chen and Chacón [2, 3, 4] designed a class of fully implicit methods that conserve charge and energy at the same time.

The Energy Conserving Semi-Implicit Method (ECSIM) [5] conserves the energy up to the iteration tolerance. It is faster than the explicit PIC methods due to the relaxed temporal and spatial resolution constraints. ECSIM is 
also more efficient than the fully explicit methods, because ECSIM does not require the particles to be involved during the iterations. Lapenta[5] demonstrated that ECSIM is about one order faster than a fully implicit PIC code for 1D problems when the same grid resolution and number of particles are used (Table 1 and Table 2 of [5]). A potential flaw of ECSIM is the lack of any mechanism ensuring the satisfaction of Gauss's law. The violation of Gauss's law may generate numerical artifacts. The electric field correction method can be easily applied to ECSIM to improve the charge conservation, but it destroys the energy conservation property, and more importantly it does not behave well for certain cases as we will demonstrate in this paper. It is also not trivial to design a current assignment algorithm to satisfy both energy conservation and charge conservation at the same time for the semi-implicit moment method.

We have successfully applied the semi-implicit PIC algorithm implemented into the iPIC3D code [20] to large-scale kinetic simulations in recent years [21, 22, 23, 24]. We found that the code may create artificial oscillations in the electric field and heat the particles numerically, which needs to be alleviated by smoothing the electric field [23, 24, 25]. Smoothing will, of course, make the solution more diffusive. ECSIM provides another option to eliminate the numerical heating by enforcing conservation of energy. We implemented the ECSIM algorithm into iPIC3D in an efficient way, which is described in section 2, but we found that ECSIM may create other numerical issues related to the violation of Gauss's law. In this paper, we introduce the novel idea to correct the particle locations at the end of each computational cycle to satisfy Gauss's law for the ECSIM algorithm. The correction keeps the energy conservation property of ECSIM because it changes neither the kinetic energy of each particle nor the electromagnetic field energy. Since there are usually at least dozens of macro-particles per cell, the displacement of each particle required to eliminate the errors in Gauss's law is not unique. In order to minimize the displacements, we apply a generalized Lagrange multiplier to minimize the total displacements of the macro-particles while satisfying Gauss's law at every grid cell. This correction is accurate but also computationally intensive. To reduce the computational cost, we also designed another two alternative approximate correction methods, which do not eliminate the error entirely, but can suppress the growth of the error effectively and are computationally less expensive. The three variants of this novel Gauss's Law satisfying Energy-Conserving Semi-Implicit Method (GL-ECSIM) are described in section 2 . 
We note that even though this particle position correction method is designed to improve the performance of ECSIM, the same idea can be easily applied to any other PIC algorithm. Correcting the particle positions instead of the electric field may be advantageous, because in general the field quantities are smoother and have less error than the particle related quantities, like charge density. Correcting the particle positions is likely to remove actual errors (compared to an exact solution), while correcting the electric field may push the errors in the particle positions into the electric field.

Besides the Gauss's law satisfaction issue, we also found ECSIM may produce short-wavelength oscillation due to the simple spatial discretization used for the electric field solver. Section 2 also discusses the modifications that are necessary to suppress the oscillations. Numerical tests in section 3 justify the necessity of improving the charge conservation property and other modifications, and demonstrate the quality of our algorithm. Finally, section 4 presents the conclusions.

\section{The Gauss's law satisfying energy-conserving semi-implicit method (GL-ECSIM)}

\subsection{The electric field solver}

GL-ECSIM is based on the Energy-Conserving Semi-Implicit Method (ECSIM) developed by Lapenta [5]. ECSIM uses a staggered grid, where the electric field is defined at cell nodes, and the magnetic field is stored at cell centers. The position and velocity of a macro-particle are staggered in time, i.e., the particle velocity is at the integer time stage and the location is at the half time stage. Lapenta [5] updates the electric field and magnetic field at the same time by an implicit solver:

$$
\begin{aligned}
& \frac{\mathbf{B}^{n+1}-\mathbf{B}^{n}}{\Delta t}=-c \nabla \times \mathbf{E}^{n+\theta} \\
& \frac{\mathbf{E}^{n+1}-\mathbf{E}^{n}}{\Delta t}=c \nabla \times \mathbf{B}^{n+\theta}-4 \pi \overline{\mathbf{J}}
\end{aligned}
$$

where $\overline{\mathbf{J}}$ is the predicted current at $n+\frac{1}{2}$ time stage, and it depends on the unknown electric field $\mathbf{E}^{n+\theta}$. The definition of current $\overline{\mathbf{J}}$ can be found in [5]. The value at time level $n+\theta$ is defined as a linear combination of the values 
at the $n$ and $n+1$ stages such that:

$$
\begin{aligned}
\mathbf{E}^{n+\theta} & =(1-\theta) \mathbf{E}^{n}+\theta \mathbf{E}^{n+1} \\
\mathbf{B}^{n+\theta} & =(1-\theta) \mathbf{B}^{n}+\theta \mathbf{B}^{n+1}
\end{aligned}
$$

Instead of solving for $\mathbf{E}^{\mathbf{n}+\mathbf{1}}$ and $\mathbf{B}^{n+1}$ at the same time, we replace $\mathbf{B}^{n+1}$ and $\mathbf{E}^{n+1}$ in eq.(5) and eq.(6) with linear combinations of $\mathbf{B}^{n}, \mathbf{B}^{n+\theta}$ and $\mathbf{E}^{n}$, $\mathbf{E}^{n+\theta}$, respectively, express $\mathbf{B}^{n+\theta}$ from eq. (5) and substitute this into eq. (6) to obtain an equation that only contains the electric field as unknowns:

$$
\mathbf{E}^{n+\theta}+\delta^{2}\left[\nabla\left(\nabla \cdot \mathbf{E}^{n+\theta}\right)-\nabla^{2} \mathbf{E}^{n+\theta}\right]=\mathbf{E}^{n}+\delta\left(\nabla \times \mathbf{B}^{n}-\frac{4 \pi}{c} \overline{\mathbf{J}}\right),
$$

where $\delta=c \theta \Delta t$, and the identity $\nabla \times \nabla \times \mathbf{E}=\nabla(\nabla \cdot \mathbf{E})-\nabla^{2} \mathbf{E}$ is used, which also holds numerically for the specific spatial discretization of the ECSIM algorithm. After $\mathbf{E}^{n+\theta}$ is obtained, the magnetic field at time level $n+1$ can be easily calculated from eq.(5). Solving eq.(9) is equivalent to solving eqs. (5) - (6) analytically. But there are some numerical advantages of solving eq.(9) instead of eq.(5) - eq.(6):

- The number of unknown variables per grid cell is reduced from 6 to 3 .

- Eq. (9) transfers two curl operators in eqs.(6) - (5) into a Laplacian and a gradient-divergence term. The Laplacian operator is diagonally dominant and helps to speed up the convergence. This transformation is proposed by Chacón and Knoll [26], and known as the 'physics-based' preconditioner.

We use the GMRES iterative scheme to solve eq.(9). The magnetic field is updated from eq.(5) after the electric field is obtained.

As it has been pointed out by Lapenta [5], the exact energy conservation can be achieved only if $\theta=0.5$ and proper spatial discretizations are used. But simulations with $\theta=0.5$ have more noise than the simulations with $\theta=1$ [5]. Our tests in section 3 confirm that simulations with $\theta=0.5$ may create numerical waves. We propose using $\theta=0.51$ instead. This choice sacrifices the energy conservation a little bit, but improves the robustness significantly. Our observations are consistent with Tanaka's work [27, 28] that pointed out that $\theta>0.5$ damps the light waves and the Langmuir oscillations in a semiimplicit PIC method that uses a temporal discretization similar to ECSIM. 


\subsection{The pseudo-current}

The ECSIM method is the further development of the iPIC3D code [20], which also solves an electric field equation similar to eq.(9). Our numerical tests show iPIC3D satisfies Gauss's law better than the ECSIM method in general, because iPIC3D incorporates a 'pseudo-current' 8] term into its electric field solver. To illustrate this point, we add a $(c \Delta t)^{2} \nabla \nabla \cdot \mathbf{E}^{n+1}$ term to both sides of eq.(15) in [20], which is the electric field equation iPIC3D solves, and re-organize it:

$$
\begin{aligned}
\mathbf{E}^{n+1}+(c \Delta t)^{2}\left[\nabla\left(\nabla \cdot \mathbf{E}^{n+1}\right)-\nabla^{2} \mathbf{E}^{n+1}\right]= & \mathbf{E}^{n}+c \Delta t\left(\nabla \times \mathbf{B}^{n}-\frac{4 \pi}{c} \overline{\mathbf{J}}\right) \\
& -(c \Delta t)^{2} \nabla\left(4 \pi \rho^{n+1}-\nabla \cdot \mathbf{E}^{n+1}\right),
\end{aligned}
$$

where $\overline{\mathbf{J}}$ is the current at half time stage, just as the current in eq. $(9)$ but it is calculated in a different way, and $\rho^{n+1}$ is the estimated net charge density at the $n+1$ stage:

$$
\begin{gathered}
\overline{\mathbf{J}}=\hat{\mathbf{J}}+\frac{\chi^{n}}{4 \pi \Delta t} \mathbf{E}^{n+1}, \\
\rho^{n+1}=\rho^{n}-\Delta t \nabla \cdot \overline{\mathbf{J}} .
\end{gathered}
$$

The definition of $\hat{\mathbf{J}}$ and $\chi^{n}$ can be found in [20]. The last two terms in eq. (10), which are the difference between the charge and the divergence of the electric field, correspond to the 'pseudo-current' and diffuse the errors away. The diffusion effect can be seen by taking the divergence of the semi-discretized equation eq. (10), and applying the equality $\nabla \times \nabla \times \mathbf{E}=\nabla(\nabla \cdot \mathbf{E})-\nabla^{2} \mathbf{E}$ and the electric charge continuity equation eq. $(12)$ :

$$
\frac{\left(\nabla \cdot \mathbf{E}^{n+1}-4 \pi \rho^{n+1}\right)-\left(\nabla \cdot \mathbf{E}^{n}-4 \pi \rho^{n}\right)}{\Delta t}=c^{2} \Delta t \nabla^{2}\left(\nabla \cdot \mathbf{E}^{n+1}-4 \pi \rho^{n+1}\right),
$$

which is a diffusion equation for the error in Gauss's law. A more detailed analysis can be found in Marder [8].

When $\theta=1$ is chosen for the ECSIM solver eq.(9), it is very similar to the iPIC3D solver eq. (10) except that there is a pseudo-current term in the iPIC3D solver and these two PIC methods use different algorithms to calculate the current $\overline{\mathbf{J}}$. The pseudo-current method can be applied to the ECSIM's electric field solver eq. (9) as well. We add the term $-\delta^{2} \nabla\left(4 \pi \rho^{n+1 / 2}-\right.$ 
$\left.\nabla \cdot \mathbf{E}^{n+\theta}\right)$ to the right-hand side of eq. $(9)$ and move the $\nabla \cdot \mathbf{E}^{n+\theta}$ term to the left-hand side to obtain:

$$
\begin{aligned}
\mathbf{E}^{n+\theta}+\delta^{2}\left[\left(1-c_{p c}\right) \nabla\left(\nabla \cdot \mathbf{E}^{n+\theta}\right)-\nabla^{2} \mathbf{E}^{n+\theta}\right]= & \mathbf{E}^{n}+\delta\left(\nabla \times \mathbf{B}^{n}-\frac{4 \pi}{c} \overline{\mathbf{J}}\right) \\
& -c_{p c} \delta^{2} \nabla\left(4 \pi \rho^{n+\frac{1}{2}}\right)
\end{aligned}
$$

where $c_{p c}$ is the coefficient of the pseudo-current. It is easy to implement this pseudo-current term, because the field $\mathbf{E}^{n+\theta}$ is already part of the field solver and the net charge $\rho^{n+\frac{1}{2}}$ can be calculated from the particles in advance. We use $\mathbf{E}^{n+\theta}$ and $\rho^{n+\frac{1}{2}}$ to form the pseudo-current term for simplicity. $\mathbf{E}^{n+\theta}$ and

$\rho^{n+\frac{1}{2}}$ are not necessarily at the same time stage unless $\theta=0.5$. In section 3 , we show that the pseudo-current scheme does not work well for the ECSIM method in general, because it ruins the energy conservation.

\subsection{Particle position correction}

The electric field correction methods, such as the 'pseudo-current' method, modify the electric field to reduce the discrepancy in Gauss's law. If most of the error in Gauss's law is due to the inaccuracy of the net charge, which comes from the particle mover, the field correction method will not work well even though Gauss's law is satisfied formally.

In this section, we introduce a new idea of displacing the particles to satisfy Gauss's law. The displacement is done at the end of each computational cycle after each particle has updated its velocity and position. Since neither the electromagnetic field nor the particle velocity are changed by the particle position correction, the energy conservation still holds. The particle position correction method can be accurate or approximate. The accurate correction need to calculate the particle displacement carefully to perfectly satisfy Gauss's law at every grid cell, while the approximate correction just moves the particles in the right direction to reduce the error in Gauss's law.

\subsubsection{The accurate correction}

In one computational cycle, the electromagnetic field is updated from $\mathbf{E}_{q}^{n}$ and $\mathbf{B}_{c}^{n}$ to $\mathbf{E}_{g}^{n+1}$ and $\mathbf{B}_{c}^{n+1}$, the particle's velocity is updated from $\mathbf{v}_{p}^{n}$ to $\mathbf{v}_{p}^{n+1}$ and the particle is moved from $\mathbf{x}_{p}^{n+\frac{1}{2}}$ to $\tilde{\mathbf{x}}_{p}^{n+\frac{3}{2}}$. We use subscripts $p, c$ and $g$ to represent particles, cell centers and cell nodes, respectively. The tilde marks the values before the correction. 
(a) $\quad b_{0}\left(\frac{x-x_{c}}{\Delta x}\right)$

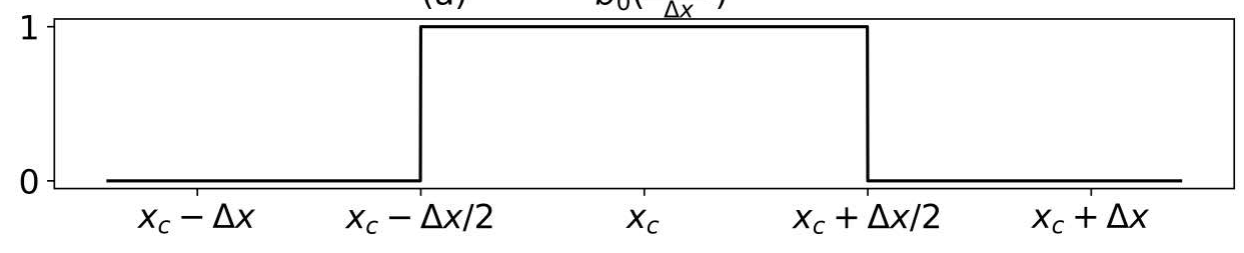

(b) $\quad b_{1}\left(\frac{x-x_{c}}{\Delta x}\right)$

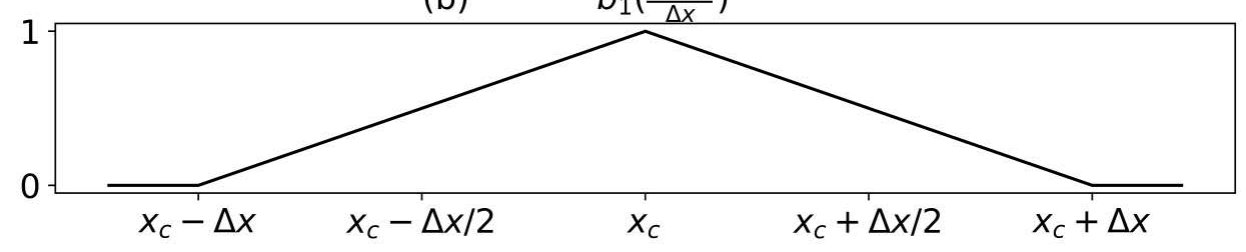

(c) $\quad b_{1}^{\prime}\left(\frac{x-x_{c}}{\Delta x}\right)$

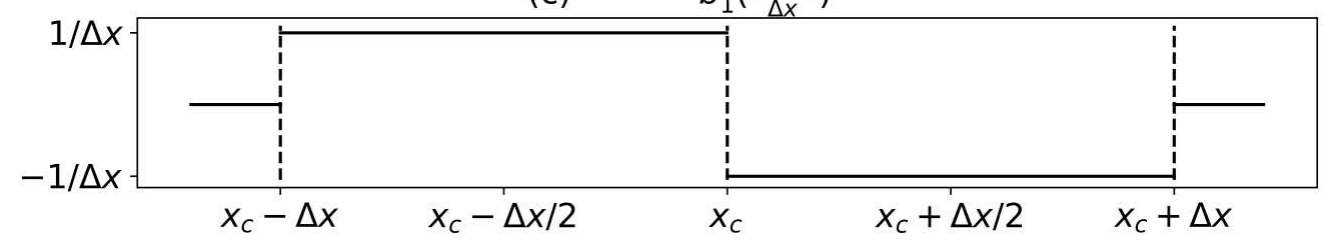

Figure 1: The B-spline functions and the derivative of $b_{1}$. The $b_{0}$ spline at the top is used in the shape function $S$ while the $b_{1}$ spline in the middle is used for the interpolation function $W$. The derivative of $b_{1}$ at the bottom is needed in the gradient of $W$. 
We use the node electric field and cell center net charge to evaluate the error in Gauss's law. The net charge density at the cell center is interpolated from particles. For example,

$$
\rho_{c}^{n+\frac{1}{2}}=\sum_{p} q_{p} W\left(\mathbf{x}_{p}^{n+\frac{1}{2}}-\mathbf{x}_{c}\right)
$$

where $\rho_{c}^{n+\frac{1}{2}}$ is the cell center net charge density at the $n+\frac{1}{2}$ time stage, $q_{p}$ is the charge of a macro-particle and $W\left(\mathbf{x}_{p}^{n+\frac{1}{2}}-\mathbf{x}_{c}\right)$ is the interpolation function, which is also known as the weight function, from the particle's location $\mathbf{x}_{p}^{n+\frac{1}{2}}$ to the cell center $\mathbf{x}_{c}$. We note that a macro-particle represents millions of physical particles that are close to each other in the phase space, and each macro-particle may carry different amounts of charge corresponding to $q_{p}$ but the charge per mass ratio is the same for all particles representing the same species (for example electrons).

At the end of one computational cycle, the particle's position and the electric field are at different stages. In order to evaluate and fix the error of Gauss's law at time stage $n+1$, we interpolate the charge density $\rho_{c}^{n+1}$ from $\rho_{c}^{n+\frac{3}{2}}$ and $\rho_{c}^{n+\frac{1}{2}}$. The goal is to add a displacement $\Delta \mathbf{x}_{p}$ to each particle's position $\tilde{\mathbf{x}}_{p}^{n+\frac{3}{2}}$ so that the density $\rho_{c}^{n+1}$ satisfies Gauss's law:

$$
\rho_{c}^{n+1}=\gamma \sum_{p} q_{p} W\left(\tilde{\mathbf{x}}_{p}^{n+\frac{3}{2}}+\Delta \mathbf{x}_{p}-\mathbf{x}_{c}\right)+(1-\gamma) \rho_{c}^{n+\frac{1}{2}}=\frac{1}{4 \pi} \nabla \cdot \mathbf{E}^{n+1},
$$

where $\gamma$ is an interpolation coefficient. When $\gamma=0.5$, the interpolation is second-order accurate. But our tests suggest that using $\gamma=0.5$ may cause numerical oscillations. Similarly to the optimal choice of the $\theta$ parameter, we find that $\gamma=0.51$ works very well. It sacrifices the accuracy slightly but eliminates the artificial oscillations. $\gamma=0.51$ is used in this paper. Our goal is to displace the particles so that the equation above is satisfied at all cell centers. This equation system is likely to be under-determined in general, because there are usually more particles (and corresponding unknown displacement vectors $\Delta \mathbf{x}_{p}$ ) than the number of cell centers (corresponding to the number of equations). The position correction can be applied to only one species (for example electrons only) or all species. In the following derivation of this accurate correction method, we assume that the correction is applied to all species. 
The displacement $\Delta \mathbf{x}_{p}$ should be small with respect to the cell size. Under the assumption of small displacements, the computation can be simplified by linearizing the interpolation function:

$$
W\left(\tilde{\mathbf{x}}_{p}^{n+\frac{3}{2}}+\Delta \mathbf{x}_{p}-\mathbf{x}_{c}\right)=W\left(\tilde{\mathbf{x}}_{p}^{n+\frac{3}{2}}-\mathbf{x}_{c}\right)+\nabla W\left(\tilde{\mathbf{x}}_{p}^{n+\frac{3}{2}}-\mathbf{x}_{c}\right) \cdot \Delta \mathbf{x}_{p}+O\left((\Delta x)^{2}\right) .
$$

In our GL-ECSIM code, we use the zeroth order B-spline function $b_{0}$ (see Figure.17) to form the 3-dimensional shape function of the macro-particles:

$$
S\left(\mathbf{x}_{p}-\mathbf{x}_{c}\right)=\frac{1}{\Delta x \Delta y \Delta z} b_{0}\left(\frac{x_{p}-x_{c}}{\Delta x}\right) b_{0}\left(\frac{y_{p}-y_{c}}{\Delta y}\right) b_{0}\left(\frac{z_{p}-z_{c}}{\Delta z}\right) .
$$

The $S$ function is a top-hat function centered around the particle with the width of the cell size. The interpolation function from a particle to a cell center is the integral of the particle's shape function over this cell, which leads to the first-order B-spline function $b_{1}$ (see Figure.1 $\mathrm{b}$ ). In a three dimensions (3D), the interpolation function is

$$
W\left(\mathbf{x}_{p}-\mathbf{x}_{c}\right)=b_{1}\left(\frac{x_{p}-x_{c}}{\Delta x}\right) b_{1}\left(\frac{y_{p}-y_{c}}{\Delta y}\right) b_{1}\left(\frac{z_{p}-z_{c}}{\Delta z}\right)
$$

The $b_{1}\left(\frac{x_{p}-x_{c}}{\Delta x}\right)$ function is differentiable with respect to $x_{p}$ when $\frac{x_{p}-x_{c}}{\Delta x} \neq$ $0, \pm 1$ (see Figure.1p):

$$
b_{1}^{\prime}\left(\frac{x_{p}-x_{c}}{\Delta x}\right)= \begin{cases}-1 / \Delta x, & \text { if } x_{c}<x_{p}<x_{c}+\Delta x \\ 1 / \Delta x, & \text { if } x_{c}-\Delta x<x_{p}<x_{c} \\ 0, & \text { if } x_{p}<x_{c}-\Delta x \text { or } x_{p}>x_{c}+\Delta x\end{cases}
$$

This spatial derivative suggests that if we move a particle toward (away from) the cell center, the interpolation weight from the particle to this cell center will increase (decrease). If the particle is so close to the cell center that the displacement $\Delta x_{p}$ makes the particle cross the cell center, we cannot predict the change of the interpolation weight from the $b_{1}$ derivative because the $b_{1}$ function is not differentiable at $b_{1}(0)$. For these particles, the linearization of eq. (17) is not valid. In practice, only a small portion of all the particles may encounter this problem when the displacement is generally small. This means that the non-differentiability will have little effect in general and the problem is getting smaller with smaller displacements.

With the spatial derivative of the $b_{1}$ function known, the gradient of the interpolation function can be obtained. For example, when $x_{c}<x_{p}<$ 
$x_{c}+\Delta x, y_{c}<y_{p}<y_{c}+\Delta y$ and $z_{c}<z_{p}<z_{c}+\Delta z$, the interpolation function is:

$$
W\left(\mathbf{x}_{p}-\mathbf{x}_{c}\right)=\frac{\left(x_{c}+\Delta x-x_{p}\right)\left(y_{c}+\Delta y-y_{p}\right)\left(z_{c}+\Delta z-z_{p}\right)}{\Delta x \Delta y \Delta z}
$$

and its gradient is:

$$
\nabla W\left(\mathbf{x}_{p}-\mathbf{x}_{c}\right)=\left(\frac{-W\left(\mathbf{x}_{p}-\mathbf{x}_{c}\right)}{x_{c}+\Delta x-x_{p}}, \frac{-W\left(\mathbf{x}_{p}-\mathbf{x}_{c}\right)}{y_{c}+\Delta y-y_{p}}, \frac{-W\left(\mathbf{x}_{p}-\mathbf{x}_{c}\right)}{z_{c}+\Delta z-z_{p}}\right) .
$$

From this example, we can see that the interpolation function is not linear and the $O\left((\Delta x)^{2}\right)$ term in eq. 17$)$ will not vanish.

We substitute eq. (17) into eq.(16) and drop the $O\left((\Delta x)^{2}\right)$ term to obtain the linearized Gauss's law constrain for a given cell center:

$$
g_{c}\left(\Delta \mathbf{x}_{p}\right):=\sum_{p} q_{p} \nabla W\left(\tilde{\mathbf{x}}_{p}^{n+\frac{3}{2}}-\mathbf{x}_{c}\right) \cdot \Delta \mathbf{x}_{p}-S_{c}=0
$$

where the constant term (independent of $\Delta \mathbf{x}_{p}$ ) is

$$
S_{c}:=\frac{1}{\gamma}\left[\frac{1}{4 \pi} \nabla \cdot \mathbf{E}^{n+1}-\left((1-\gamma) \rho_{c}^{n+\frac{1}{2}}+\gamma \sum_{p} q_{p} W\left(\tilde{\mathbf{x}}_{p}^{n+\frac{3}{2}}-\mathbf{x}_{c}\right)\right)\right]
$$

Both $g_{c}\left(\Delta \mathbf{x}_{p}\right)$ and $S_{c}$ are defined at every cell center. To find a solution for the under-determined equations above while minimizing the displacements, we use the Lagrange multiplier method. The function we are trying to minimize is defined as

$$
f\left(\Delta \mathbf{x}_{p}\right)=\sum_{p} \frac{1}{2}\left(\Delta \mathbf{x}_{p}\right)^{2}\left|q_{p}\right|^{\alpha}
$$

where $\alpha$ is a non-negative exponent to be specified later. Our goal is to minimize the function $f\left(\Delta \mathbf{x}_{p}\right)$ provided that eq. 23) is satisfied for each cell center. The Lagrange function is:

$$
\begin{aligned}
L\left(\Delta \mathbf{x}_{p}, \lambda_{c}\right) & =f\left(\Delta \mathbf{x}_{p}\right)-\sum_{c} \lambda_{c} g_{c}\left(\Delta \mathbf{x}_{p}\right) \\
& =\sum_{p} \frac{1}{2}\left(\Delta \mathbf{x}_{p}\right)^{2}\left|q_{p}\right|^{\alpha}-\sum_{c} \lambda_{c}\left[\sum_{p} q_{p} \nabla W\left(\tilde{\mathbf{x}}_{p}^{n+\frac{3}{2}}-\mathbf{x}_{c}\right) \cdot \Delta \mathbf{x}_{p}-S_{c}\right]
\end{aligned}
$$


where $\lambda_{c}$ are the Lagrange multiplier for all the cell centers. The function $f$ reaches a local extrema if the Lagrange function's partial derivatives with respect to the displacements $\Delta \mathbf{x}_{p}$ and the Lagrange multipliers $\lambda_{c}$ are all zero:

$$
\begin{array}{r}
\frac{\partial L}{\partial \lambda_{c}}=g_{c}\left(\Delta \mathbf{x}_{p}\right)=\sum_{p} q_{p} \nabla W\left(\tilde{\mathbf{x}}_{p}^{n+\frac{3}{2}}-\mathbf{x}_{c}\right) \cdot \Delta \mathbf{x}_{p}-S_{c}=0 \\
\frac{\partial L}{\partial \Delta \mathbf{x}_{p}}=\Delta \mathbf{x}_{p}\left|q_{p}\right|^{\alpha}-\sum_{c} \lambda_{c} q_{p} \nabla W\left(\tilde{\mathbf{x}}_{p}^{n+\frac{3}{2}}-\mathbf{x}_{c}\right)=0 .
\end{array}
$$

Thanks to the linearization, the displacement of each particle can be easily expressed as a function of $\lambda_{c}$ by solving eq. (28):

$$
\Delta \mathbf{x}_{p}=\sum_{c} \lambda_{c}\left|q_{p}\right|^{-\alpha} q_{p} \nabla W\left(\tilde{\mathbf{x}}_{p}^{n+\frac{3}{2}}-\mathbf{x}_{c}\right)
$$

and substituted into eq. 27) to obtain a linear system of equations that only contains $\lambda_{c}$ as unknowns:

$$
\frac{\partial L}{\partial \lambda_{c}}=\sum_{p} q_{p} \nabla W\left(\tilde{\mathbf{x}}_{p}^{n+\frac{3}{2}}-\mathbf{x}_{c}\right) \cdot\left[\left|q_{p}\right|^{-\alpha} q_{p} \sum_{c^{\prime}} \lambda_{c^{\prime}} \nabla W\left(\tilde{\mathbf{x}}_{p}^{n+\frac{3}{2}}-\mathbf{x}_{c^{\prime}}\right)\right]-S_{c}=0
$$

We note that this is an equation for cell center $c$ so we introduced $c^{\prime}$ for the summation. After exchanging the order of the two summations for $c^{\prime}$ and $p$, we obtain

$$
\sum_{c^{\prime}} M_{c c^{\prime}} \lambda_{c^{\prime}}=S_{c}
$$

where the matrix element $M_{c c^{\prime}}$ is defined as:

$$
M_{c c^{\prime}}:=\sum_{p}\left|q_{p}\right|^{2-\alpha} \nabla W\left(\tilde{\mathbf{x}}_{p}^{n+\frac{3}{2}}-\mathbf{x}_{c}\right) \cdot \nabla W\left(\tilde{\mathbf{x}}_{p}^{n+\frac{3}{2}}-\mathbf{x}_{c^{\prime}}\right) .
$$

Once the 'mass matrix' $M$ is calculated, the Lagrange multipliers $\lambda_{c}$ can be obtained by solving the linear system eq. (31), then we can calculate the particle displacement $\Delta \mathbf{x}_{p}$ from eq. 28 and add the displacements to $\tilde{\mathbf{x}}_{p}^{n+\frac{3}{2}}$ to obtain the corrected particle positions:

$$
\mathbf{x}_{p}^{n+\frac{3}{2}}=\tilde{\mathbf{x}}_{p}^{n+\frac{3}{2}}+\Delta \mathbf{x}_{p}
$$


We use the GMRES iterative method to solve eq. (31).

Since the $O\left((\Delta x)^{2}\right)$ term is not zero in eq. 117), there is still an error of $O\left((\Delta x)^{2}\right)$ in Gauss's law (see eq. 16) after the correction. To further minimize the error, we can repeat the correction several times. The particle displacement decreases when we repeat the correction, so it also helps to reduce the influence of the singularity in the $b_{1}$ derivative (see eq. 200 ). In section 3, we show that after three corrections, the error in Gauss's law reduces to a very small value.

We can now determine the most sensible value for the $\alpha$ exponent introduced in eq. 25). If two particles of the same species overlap with each other before the correction, it is natural to correct them with the same displacement, i.e., their displacements $\Delta \mathbf{x}_{p}$ should not depend on the particle's charge $q_{p}$. According to eq. (29) this will hold if we set $\alpha=1$, which is the value used in all simulations in this paper. When $\alpha=1$, eq.25 implies that the Lagrange function minimizes the sum of $\left|q_{p}\right|\left(\Delta x_{p}\right)^{2}$ over the particles.

We assumed that all species are corrected above, but we have the freedom to correct one species only. In that case only the particles that require correction are looped through to calculate the matrix $M$ (see eq.(32) ) and the displacement $\Delta \mathbf{x}_{p}$ (see eq. $(29)$ ), which are the two most expensive parts of one correction cycle. So it is better to correct only one species in terms of computational efficiency. We find that correcting the lightest species (typically electrons) only is a reasonable choice in practice.

\subsubsection{The approximate global correction}

The accurate correction reduces the error in Gauss's law to the iterative tolerance level. But it requires looping through particles to calculate the matrix $M$ (see eq. (32)). This step is computationally expensive. If the goal is to suppress the growth of the error in Gauss's law instead of eliminating it entirely, the calculation of the matrix $M$ can be avoided.

Boris' electric field correction method solves the following Poisson equation of the scalar function $\phi$ defined at cell centers:

$$
\nabla^{2} \phi=\nabla \cdot \tilde{\mathbf{E}}^{n+1}-4 \pi \tilde{\rho}_{c}^{n+1},
$$

where $\tilde{\mathbf{E}}$ and $\tilde{\rho}_{c}$ are the uncorrected electric field and charge density at the cell center. After $\phi$ is obtained, the electric field is corrected to satisfy Gauss's law:

$$
\mathbf{E}^{n+1}=\tilde{\mathbf{E}}^{n+1}-\nabla \phi .
$$


Instead of correcting the electric field, we design an analogous algorithm that corrects the particle positions. Similar to the Boris field correction, we solve the Poisson equation (34) first with the GMRES scheme. The charge density is interpolated as

$$
\tilde{\rho}_{c}^{n+1}=\gamma \tilde{\rho}_{c}^{n+\frac{3}{2}}+(1-\gamma) \rho_{c}^{n+\frac{1}{2}}
$$

where the tilde represents the charge density before position correction and $\gamma=0.51$ is an interpolation coefficient as in eq.(16). If we could find displacements $\Delta \mathbf{x}_{p}$ for each particle so that

$$
\rho^{n+\frac{3}{2}}\left(\tilde{\mathbf{x}}_{p}^{n+\frac{3}{2}}+\Delta \mathbf{x}_{p}\right)=\tilde{\rho}^{n+\frac{3}{2}}\left(\tilde{\mathbf{x}}_{p}^{n+\frac{3}{2}}\right)+\frac{1}{4 \pi \gamma} \nabla^{2} \phi,
$$

it is easy to show that the corrected and time interpolated charge density $\rho_{c}^{n+1}$ and the original electric field $\mathbf{E}^{n+1}=\tilde{\mathbf{E}}^{n+1}$ will satisfy Gauss's law. So, the goal is to find the displacements $\Delta \mathbf{x}_{p}$ that satisfy the above equation where $\phi$ is given by eq. 34 .

When we add the displacement $\Delta \mathbf{x}_{p}$ to a particle, it is equivalent to add a 'virtual current' $\mathbf{j}_{v}$ for a 'virtual time' $\Delta t_{v}$ to change the charge density from $\tilde{\rho}^{n+\frac{3}{2}}\left(\tilde{\mathbf{x}}_{p}^{n+\frac{3}{2}}\right)$ to $\rho^{n+\frac{3}{2}}\left(\tilde{\mathbf{x}}_{p}^{n+\frac{3}{2}}+\Delta \mathbf{x}_{p}\right)$. The charge conservation equation describes how the 'virtual current' changes the charge density:

$$
\rho^{n+\frac{3}{2}}\left(\tilde{\mathbf{x}}_{p}^{n+\frac{3}{2}}+\Delta \mathbf{x}_{p}\right)-\tilde{\rho}^{n+\frac{3}{2}}\left(\tilde{\mathbf{x}}_{p}^{n+\frac{3}{2}}\right)=\nabla \cdot\left(\Delta t_{v} \mathbf{j}_{v}\right)+\text { discretization error. }
$$

Combining eq.(38) and eq.(37), we obtain the equation for the $\Delta t_{v} \mathbf{j}_{v}$ term:

$$
\Delta t_{v} \mathbf{j}_{v}=\frac{1}{4 \pi \gamma} \nabla \phi+\text { discretization error. }
$$

For the sake of simplicity, we only displace the electrons or the lightest species to create the 'virtual current'. For a given position $\mathbf{x}_{p}$, if we displace the surrounding electrons by $\Delta \mathbf{x}_{p}$, it will generate a 'virtual current':

$$
\left(\Delta t_{v} \mathbf{j}_{v}\right)_{p}=\rho_{e, p} \Delta \mathbf{x}_{p} \approx \rho_{e, g} \Delta \mathbf{x}_{p}
$$

where $\rho_{e, p}, \rho_{e, g}$ are the electron charge densities at $\mathbf{x}_{p}$ and its closest node, respectively. Combining the two equations above and ignoring the discretization errors, the displacement $\Delta \mathbf{x}_{p}$ is obtained as

$$
\Delta \mathbf{x}_{p}=\frac{1}{4 \pi \gamma \rho_{e, g}} \nabla \phi
$$




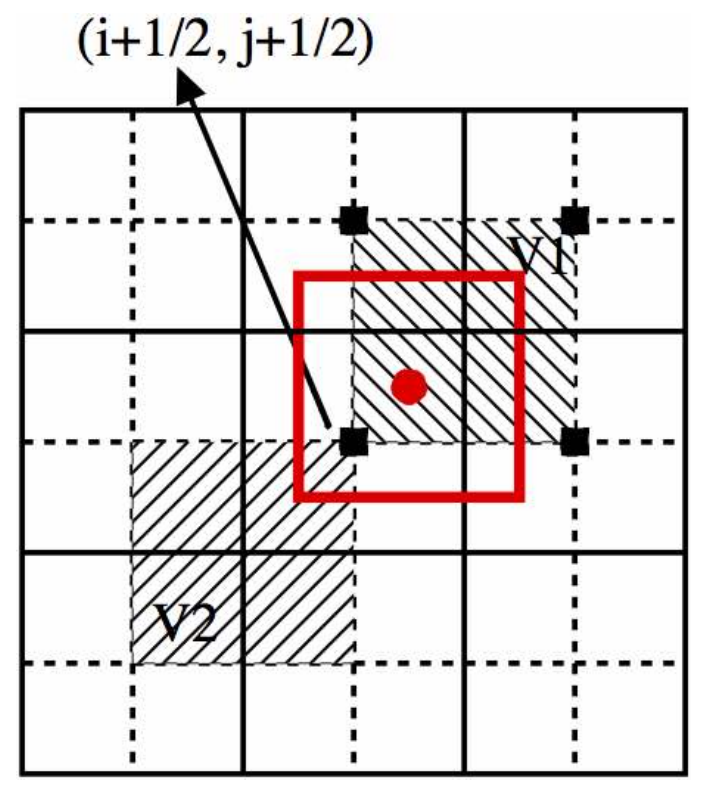

Figure 2: The black solid lines represent the cell edges. The black squares are the cell centers. The red square represents the shape function $S_{p}$ of a macro-particle with its position $\mathbf{x}_{p}$ marked by the red circle. The two shaded squares are two complementary volumes (node-centered volumes) V1 and V2.

This global approximate correction method solves a Poisson's equation to distribute the 'virtual current' globally. It does not eliminate the error in Gauss's law exactly, but it pushes the particles toward the direction to reduce the error. To avoid potential overshoot, we can apply partial correction only:

$$
\Delta \mathbf{x}_{p}=\frac{\epsilon}{4 \pi \gamma \rho_{e, g}} \nabla \phi
$$

where $\epsilon$ is a constant between 0 and 1 . We use $\epsilon=0.9$ in practice. The spatial discretization is described in the section 2.3.3.

\subsubsection{The approximate local correction}

The approximate global correction method described in the previous section needs to solve a Poisson equation. Its computational cost is negligible within our GL-ECSIM scheme. But the cost may not be acceptable for an explicit PIC algorithm. To avoid solving the Poisson equation, we introduce a local correction method. 
Again, we only correct the electrons for simplicity. We calculate the relative error at each cell center first:

$$
r_{c}=\frac{\tilde{\rho}_{c}^{n+1}-\nabla \cdot \mathbf{E}^{n+1} /(4 \pi)}{\gamma \rho_{e, c}}
$$

where $\tilde{\rho}_{c}^{n+1}$ is obtained from eq. (36). The displacement $\Delta \mathbf{x}_{p}$ for a particle at $\mathbf{x}_{p}$ is calculated from

$$
\left(\Delta x_{p} / \Delta x, \Delta y_{p} / \Delta y, \Delta z_{p} / \Delta z\right)=-\epsilon\left(\frac{\Delta x}{2} \frac{\partial r_{c}}{\partial x}, \frac{\Delta y}{2} \frac{\partial r_{c}}{\partial y}, \frac{\Delta z}{2} \frac{\partial r_{c}}{\partial z}\right)_{p}
$$

where the right-hand side is the difference of the relative error $r_{c}$ in the three directions, $\Delta x, \Delta y$ and $\Delta z$ are the cell sizes, and $\epsilon$ is the correction ratio between 0 and 1 . The difference of the relative error $r_{c}$ indicates the direction to move particles. As an example, let us consider a uniform 1D simulation with a pair of electron and ion at each node at time stage $n+\frac{1}{2}$. Assume the cell size is 1 , each ion macro-particle has charge $q_{i}$ and each electron has charge $q_{e}=-q_{i}$, so the cell center electron charge density is $\rho_{e, c}=q_{e}$ and the net charge at $n+\frac{1}{2}$ stage is zero. We assume the electric field at $n+1$ is also zero. If an electron macro-particle at the cell center $x_{i}$ is misplaced at $x_{i}+0.1 \Delta x$ at $n+\frac{3}{2}$ stage and other particles do not move, the electron charge at cell centers $x_{i-1 / 2}$ and $x_{i+1 / 2}$ will become $0.9 q_{e}$ and $1.1 q_{e}$, respectively. The relative errors $r_{c}$ at $x_{i-1 / 2}$ and $x_{i+1 / 2}$ are $\frac{-0.1}{0.9} \approx-0.11$ and $\frac{0.1}{1.1} \approx 0.091$, respectively. Based on the correction formula above, the correction for this electron particle is $\Delta x_{p} / \Delta x=-\frac{\Delta x}{2} \frac{\partial r_{c}}{\partial x} \approx-(0.091+0.11) / 2 \approx-0.1$ when $\epsilon=1$, which means the electron at $x_{i}+0.1 \Delta x$ will be moved back to $x_{i}$. For this simple example, $\epsilon=1$ cancels the error almost perfectly.

Figure 2 shows a two-dimensional example. Among the 4 cell centers around the particle in the figure, the smallest index cell center is $\left(i+\frac{1}{2}, j+\frac{1}{2}\right)$. Based on the relative errors at these 4 cell centers, this particle will move toward or away from the cell center $\left(i+\frac{1}{2}, j+\frac{1}{2}\right)$. However, the information in the complementary volume $\mathrm{V} 2$ has no influence on this particle although particles inside V2 also contribute to cell center $\left(i+\frac{1}{2}, j+\frac{1}{2}\right)$. Due to the locality of this correction method, it is impossible to find a correction ratio $\epsilon$ to eliminate the error accurately in general. A large $\epsilon$ can lead to overshoots easily, while a small $\epsilon$ may not be sufficient to suppress the growth of the error. Our tests suggest that $\epsilon=0.5$ reaches a reasonable balance between the effectiveness and robustness, and it is used in the following numerical tests. 
We use Figure 2 to illustrate the calculation of the spatial derivatives in eq. (44) and eq. (42). Assume the particle is at $\left(x_{p}, y_{p}\right)$ and we need to calculate $\frac{\partial r_{c}}{\partial x}$. We interpolate $r_{x_{i+1 / 2}, y_{p}}\left(r_{x_{i+3 / 2}, y_{p}}\right)$ from $r_{x_{i+1 / 2}, y_{i+1 / 2}}$ and $r_{x_{i+1 / 2}, y_{i+3 / 2}}\left(r_{x_{i+3 / 2}, y_{i+1 / 2}}\right.$ and $\left.r_{x_{i+3 / 2}, y_{i+3 / 2}}\right)$ first. Then the spatial derivative is obtained by $\frac{\partial r_{c}}{\partial x}=\left(r_{x_{i+3 / 2}, y_{p}}-r_{x_{i+1 / 2}, y_{p}}\right) / \Delta x$.

\subsubsection{Limiting the displacement}

All the three correction methods described above assume that if a particle moves toward (away from) a cell center, its charge contribution to this center would increase (decrease). This assumption is true only when the particle center does not cross the complementary volume boundaries. When the displacement is small, there are not too many particles violating this assumption and the correction methods work well. However, in the region where the plasma is rarefied or the numerical error in Gauss's law is large, the displacement can be large compared to the cell size. To fix this problem, we limit the displacement with the following simple algorithm:

$$
\Delta \mathbf{x}_{p}^{n e w}=\min \left(1, c_{0} \frac{\Delta x}{\left|\Delta \mathbf{x}_{p}\right|}\right) \Delta \mathbf{x}_{p}
$$

where $\Delta \mathbf{x}_{p}$ the particle displacement calculated by one of the correction methods, $\Delta x$ is the cell size in the $\mathrm{x}$-direction, and $c_{0}$ is the maximum allowed relative displacement. We use $c_{0}=0.1$ for the simulations.

\subsection{Spatial discretization}

The spatial discretization of the semi-discretized equations eq.(14) and eq.(5) on a uniform Cartesian grid can be done following the iPIC3D convention. Since $\mathbf{E}$ and $\mathbf{B}$ are staggered in space, we need first-order derivatives from cell centers to nodes and from nodes to cell centers, and second-order derivatives from nodes to nodes. The node-to-node second-order derivatives can be obtained in two steps: first calculate the node-to-center first-order derivatives and then calculate the center-to-node derivatives of these firstorder derivatives. Each cell center (node) first-order derivative is calculated by averaging the 4 nodes (centers) in the transverse directions and then taking the difference between the two averaged values along the direction of the derivative. For example, the cell centered first-order derivative of $E_{x}$ in the $x$ direction is calculated as

$$
\left.\frac{\partial E_{x}}{\partial x}\right|_{i+\frac{1}{2}, j+\frac{1}{2}, k+\frac{1}{2}}=\frac{1}{\Delta x} \sum_{l, m=0}^{l, m=1} \frac{1}{4}\left(E_{x, i+1, j+l, k+m}-E_{x, i, j+l, k+m}\right)
$$


where the integer indices $\mathrm{i}, \mathrm{j}$ and $\mathrm{k}$ represent the cell nodes while the half indices represent the cell centers. All the spatial derivatives in eq. (14) can be calculated based on this rule. We note that not all spatial discretizations satisfy the identities needed for energy conservation [5] but, fortunately, the discretization described above does. It also satisfies the identity $\nabla \times \nabla \times=$ $(\nabla \nabla \cdot)-\nabla^{2}$ used in deriving eq. (9).

This finite difference algorithm uses as few neighbors as possible while maintaining symmetric discrete formulas that satisfy the various identities. It is quite optimal and it behaves well for most of our simulations. But spurious short-wavelength oscillations with wavelength of $\sim 2$ cells may occur with this compact discretization for some simulations. We found that using an extended stencil for part of the the spatial discretization of $\nabla \cdot \mathbf{E}^{n+\theta}$ in eq. (9) helps to suppress these oscillations. We take $\partial E_{x} / \partial x$ at the cell center as an example to define the difference formula with an extended stencil:

$$
\left.\frac{\partial E_{x}}{\partial x}\right|_{i+\frac{1}{2}, j+\frac{1}{2}, k+\frac{1}{2}}=\frac{1}{2 \Delta x} \sum_{l, m=-1}^{l, m=1} \frac{1}{9}\left(E_{x, i+\frac{3}{2}, j+\frac{1}{2}+l, k+\frac{1}{2}+m}-E_{x, i-\frac{3}{2}, j+\frac{1}{2}+l, k+\frac{1}{2}+m}\right)
$$

where the cell center electric field values, such as $E_{x, i+\frac{3}{2}, j+\frac{1}{2}, k+\frac{1}{2}}$, are averaged form the nearby 8 nodes. We denote the divergence calculated on the extended stencil shown by eq.(47) as $\nabla^{\prime} \cdot \mathbf{E}^{n+\theta}$, while $\nabla \cdot \mathbf{E}^{n+\theta}$ represents the usual compact discretization of eq. 46. The difference of these two divergence operators can be used to diffuse the oscillatory errors related to the $\nabla \nabla \cdot \mathbf{E}$ term. Using a linear combination of $\nabla^{\prime} \cdot \mathbf{E}^{n+\theta}$ and $\nabla \cdot \mathbf{E}^{n+\theta}$, the electric field equation becomes:

$$
\begin{array}{r}
\left.\mathbf{E}^{n+\theta}+\delta^{2}\left[\nabla\left(c_{c p t} \nabla \cdot \mathbf{E}^{n+\theta}+\left(1-c_{c p t}\right) \nabla^{\prime} \cdot \mathbf{E}^{n+\theta}\right)\right)-\nabla^{2} \mathbf{E}^{n+\theta}\right]= \\
\mathbf{E}^{n}+\delta\left(\nabla \times \mathbf{B}^{n}-\frac{4 \pi}{c} \overline{\mathbf{J}}\right),
\end{array}
$$

where the coefficient $c_{c p t}$ is the fraction of the divergence calculated with the compact derivative.

We illustrate the smoothing effect of using the extended stencil for the divergence operator with a 1D example. Let us assume that there is charge separation in a $1 \mathrm{D}$ simulation along the $\mathrm{x}$-direction that generates a variation in the $E_{x}$ component with a short wavelength. Since $\nabla \times \mathbf{E}=0$ for this case, the $\nabla\left(\nabla \cdot \mathbf{E}^{n+\theta}\right)$ and $\nabla^{2} \mathbf{E}^{n+\theta}$ terms cancel each other both analytically and 
numerically when the compact derivatives are applied. However, if $c_{c p t}$ is less than 1 so that the extended stencil derivative $\nabla^{\prime}$. is also used, then in effect we add

$$
\delta^{2}\left(1-c_{c p t}\right) \nabla\left(\nabla-\nabla^{\prime}\right) E_{x}^{n+\theta}
$$

to the right hand side of the original equation (9). The leading term in the

Taylor expansion will be a fourth derivative $-\delta^{2}\left(1-c_{c p t}\right) \frac{\Delta x^{2}}{2} \frac{\partial^{4} E_{x}}{\partial x^{4}}$ since the third derivative has zero coefficient due to the symmetry of the discrete divergence and gradient operators. This operator has a net effect of smoothing the short-wavelength oscillations in $E_{x}$.

We remark that when $c_{c p t}$ is not 1, i.e., the extended stencil divergence of the electric field is used, the total energy is not exactly conserved any more. In section 3, we are going to show that simulations with $c_{c p t}=0.9$ suppress the oscillations while still conserve energy reasonably well.

\section{Numerical tests}

This section presents three numerical tests to demonstrate the performance of the GL-ECSIM algorithm. The two-dimensional (2D) magnetosphere simulation and the $2 \mathrm{D}$ reconnection test show the improvement of the GL-ECSIM scheme compared to iPIC3D and the original ECSIM algorithm. The 1D Weibel instability test demonstrates that the particle position correction step does not change the physics.

Table 1 shows 9 different parameter combinations for the tests. We performed simulations with iPIC3D, the original ECSIM and GL-ECSIM. For the original ECSIM, the role of $\theta$ is studied (ECSIM-1 and ECSIM-2), and we show that Marder's pseudo-current method [8] does no work well (ECSIM3). For GL-ECSIM, we show that the extended stencil spatial discretization helps to suppress the short-wavelength oscillations by comparing GL-ECSIM1 and GL-ECSIM-2, and we also compare different particle position correction methods (GL-ECSIM-2 to GL-ECSIM-5).

In this test section, we set the electric field solver tolerance to be $10^{-6}$. For the accurate correction method, the correction procedure is repeated three times per computational cycle. A iteration tolerance of 0.01 and a maximum iteration number of 20 are used for the linear equation systems of the correction methods. We have not implemented any preconditioner for the iterative solver, so the linear equations may not be able to converge to the tolerance 0.01 within 20 iterations. But the correction methods still work well as the following tests demonstrate. 
Table 1: Simulation parameters and the normalized wall time for the $2 \mathrm{D}$ reconnection simulations. In the particle correction method column, 'accurate', 'approximate-global' and 'approximate-local' represent three methods described in section 2.3. and 'all' indicates that the correction method is applied to all species, otherwise the correction is applied to electrons only. $c_{c p t}$ is the coefficient of the compact $\nabla \cdot \mathbf{E}$ discretization. $c_{p c}$ is the coefficient of the pseudo-current term. The 2D magnetic reconnection (MR) simulation wall time is normalized by the iPIC3D wall time.

\begin{tabular}{cccccc}
\hline Simulation ID & $\theta$ & Correction method & $c_{c p t}$ & $c_{p c}$ & MR wall time \\
\hline iPIC3D & 1.0 & N/A & N/A & 1.0 & 1.0 \\
ECSIM-1 & 0.5 & N/A & 1 & 0 & 1.8 \\
ECSIM-2 & 0.51 & N/A & 1 & 0 & 1.8 \\
ECSIM-3 & 0.51 & N/A & 1 & 0.1 & N/A \\
GL-ECSIM-1 & 0.51 & accurate & 1 & 0 & 2.6 \\
GL-ECSIM-2 & 0.51 & accurate & 0.9 & 0 & 2.6 \\
GL-ECSIM-3 & 0.51 & accurate-all & 0.9 & 0 & 2.9 \\
GL-ECSIM-4 & 0.51 & approximate-global & 0.9 & 0 & 2.1 \\
GL-ECSIM-5 & 0.51 & approximate-local & 0.9 & 0 & 2.0 \\
\hline
\end{tabular}

\subsection{Two-dimensional magnetosphere simulation}

The numerical modeling of the 3D magnetosphere has been the original motivation for us to develop the GL-ECSIM method. Here we use a 2D magnetosphere simulation to show the problems we encountered with iPIC3D and ECSIM, and also to demonstrate that GL-ECSIM cures these issues.

In the 2D Earth's magnetosphere simulation, we solve the ideal MHD equations with a separate electron equation to capture the global structure of the 2D magnetosphere. After a steady solution is obtained, we use the embedded PIC model to cover Earth's dayside magnetopause. The MHD code and the PIC code are two-way coupled. More details about the MHDEPIC algorithm can be found in [21, 23]

The $2 \mathrm{D}$ simulation domain extends from $x=-480 R_{E}$ to $x=32 R_{E}$ and $y=-128 R_{E}$ to $y=128 R_{E}$, where $R_{E}=6380 \mathrm{~km}$ is Earth's radius. The intrinsic magnetic field is represented by a $2 \mathrm{D}$ line dipole with magnetic field strength -3110 nT at the magnetic equator. The dipole is aligned with the $Y$ axis. The field strength of the $2 \mathrm{D}$ dipole is chosen so that the magnetopause forms at about the same distance $\left(\approx 10 R_{E}\right)$ as in reality. The inner boundary condition is set at $r=2.5 R_{E}$ with a fixed plasma density $10 \mathrm{amu} / \mathrm{cc}$ and zero plasma velocity. The external magnetic field (total field minus the intrinsic 
dipole) and the ion and electron pressures have zero gradient inner boundary conditions. The solar wind enters the simulation domain from the $+x$ direction with mass density $\rho_{\text {mass }}=5 \mathrm{amu} / \mathrm{cc}$, electron pressure $p_{e}=0.0124 \mathrm{nP}$, ion pressure $p_{i}=0.0062 \mathrm{nP}$, plasma velocity $\mathbf{u}=[-400,0,0] \mathrm{km} / \mathrm{s}$, and magnetic field $\mathbf{B}=[-0.1,-0.5,0] \mathrm{nT}$. Figure 3 shows the ion pressure in part of the simulation domain. After the MHD code reaches a steady state, the embedded PIC model is used to simulate the dayside reconnection region. The PIC region covers $6 R_{E}<x<12 R_{E}$ and $-6 R_{E}<y<6 R_{E}$ shown by the black box in Figure 3 . The ion mass-charge ratio $m_{i} / q_{i}$ is set to be 32 times larger than the ratio of a proton so that the ion inertial length $d_{i}$ is about $0.27 R_{E}$ in the magnetosheath (see [24] for more detail on the scaling). A reduced ion-electron mass ratio $m_{i} / m_{e}=100$ is used so that the electron skin depth $d_{e}$ is about $d_{i} / 10 \approx 0.027 R_{E}$. The PIC code resolution is $1 / 32 R_{E}$, so that there are about 10 cells per ion inertial length or 1 cell per electron skin depth. 400 macro-particles per cell per species are used. The time step is fixed to be $\Delta t=0.05 \mathrm{~s}$ unless otherwise specified, and the corresponding $\mathrm{CFL}$ number $\mathrm{CFL}=\max \left(v_{x, e, t h}^{\max } / \Delta x, v_{y, e, t h}^{\max } / \Delta y, v_{z, e, t h}^{\max } / \Delta z\right) \Delta t$ is about 0.25 , where $v_{e, t h}^{\max }$ is the maximum electron thermal velocity component. A reduced speed of light $c=3000 \mathrm{~km} / \mathrm{s}$ is used.

Figure 4 compares the electric field component $E_{x}$ inside the PIC domain at $t=400 \mathrm{~s}$ for iPIC3D, ECSIM with $\theta=0.5$ (ECSIM-1), ECSIM with $\theta=0.51$ (ECSIM-2), and ECSIM with $\theta=0.51$ and the pseudo-current term (ECSIM-3). iPIC3D produces short-wavelength oscillations in the magnetosphere. Our numerical tests show its wavelength is proportional to the cell size, so the oscillations can not be physical. The oscillations can be reduced by smoothing the electric field after each update [24]. ECSIM-1 and ECSIM-2 successfully suppress the magnetosphere oscillations, but there are some spurious small scale oscillations near the magnetopause and around the edge of a plasmoid. The generation of these structures is related to the violation of Gauss's law. ECSIM-3 tries to satisfy Gauss's law better by incorporating the pseudo-current term, however, it creates oscillations in the magnetosphere just as iPIC3D does. ECSIM-2 produces smoother electric field profile in the magnetosheath than ECSIM-1. To demonstrate the role of $\theta$, we performed two more simulations for ECSIM-1 and ECSIM-2 with $\Delta t=0.1 \mathrm{~s}$ (corresponding to $\mathrm{CFL} \approx 0.5$ ) instead of $\Delta t=0.05 \mathrm{~s}$ and show the results in Figure 5. ECSIM-1 generates wave-like structures in the magnetosheath, while the result of ECSIM-2 is still clean. Because of the significant improvement from $\theta=0.5$ to $\theta=0.51$, we use $\theta=0.51$ as our default value 
in practice.

Figure 6 and Figure 7 show the importance of satisfying Gauss's law and compare different particle position correction methods. We define the error in Gauss's law as $\nabla \cdot \mathbf{E}^{n+1} /(4 \pi)-\rho_{c}^{n+1}$. For ECSIM-2, the net charge density and the error are the same order, which suggests Gauss's law is already dramatically violated. After the accurate correction method is applied to electrons to fix the Gauss's law error (GL-ECSIM-1), the error reduces to about $10^{-1}[\mathrm{nT} / \mathrm{s}]$, which is about 5 orders smaller than the net charge density. GL-ECSIM-1 also eliminates most of the small scale structures in ECSIM-2, such as the $E_{x}$ oscillations near the edge of a plasmoid in the magnetosheath, but GL-ECSIM-1 produces significant short-wavelength oscillations near the magnetopause at the magnetosphere side in the $E_{x}$ and net charge density $\rho_{c}$ profiles. By using the extended stencil $\nabla \cdot \mathbf{E}$ spatial discretization (GL-ECSIM-2), these spurious oscillations are suppressed. Applying the accurate correction to all the species (GL-ECSIM-3) also obtain small error and smooth solution. The approximate global correction (GLECSIM-4) and approximate local correction (GL-ECSIM-5) can not perfectly eliminate the error in Gauss's law, and the errors are about $10^{3}[\mathrm{nT} / \mathrm{s}]$, which is 10 times smaller than the net charge $\rho_{c}$. Although the errors in GL-ECSIM4 and GL-ECSIM-5 with the approximate correction are much larger than the errors in GL-ECSIM-2 and GL-ECSIM-3 employing the accurate correction, these four simulations produce results of similar quality.

For this 2D magnetosphere test with the numerical parameters described above, the typical maximum particle displacement for GL-ECSIM-2 that corrects the electron particle positions only is about $4.0 \%$ of the cell size after the first linear solve, $0.2 \%$ after the second, and $0.03 \%$ after the final third solve which is the end of the non-linear correction. When both electron and proton particle positions are corrected (GL-ECSIM-3), the corrections are half of these values. For the approximate correction methods (GL-ECSIM-4 and GL-ECSIM-5) the typical correction is about $2 \%$ of the cell size.

These tests demonstrate GL-ECSIM method is more robust and accurate than iPIC3D and also the original ECSIM for a challenging problem. Since the pseudo-current method does not work well in general, $\theta=0.51$ is more robust than $\theta=0.5$, and the extended stencil discretization of the $\nabla \cdot \mathbf{E}$ helps to suppress spurious oscillations, we will ignore the pseudo-current method, use $\theta=0.51$ and the extended stencil discretization with $c_{c p t}=0.9$ as default in the following tests. 


\subsection{Two-dimensional double-current-sheet magnetic reconnection}

The two-dimensional magnetic reconnection problem is widely used to test plasma simulation codes. The double-current-sheet setup allows periodic boundary conditions for both directions. Here we use a setup based on the GEM-challenge [29].

The initial condition is set to satisfy the fluid force balance for both electrons and ions [30]. The simulation domain is $-12.8<x<12.8$ and $-6.4<y<6.4$ in normalized CGS unit. The speed of light is set to be $c=1$. The ion density is uniform and $n_{i}=0.0975$. The ion plasma frequency is $\omega_{p i}=\sqrt{\frac{4 \pi n_{i} e^{2}}{m_{i}}}=1.107$ and the ion inertial length $d_{i}=c / \omega_{p i}=0.903$ since $m_{i}=1$ and $q_{i}=-q_{e}=1$. A reduced ion-electron mass ratio $m_{i} / m_{e}=25$ is used, so the electron skin depth is about $d_{e}=d_{i} / 5=0.18$. Initially, there is no charge separation, $n_{e}=n_{i}$, and the electric field is $\mathbf{E}=0$.

The background magnetic filed is

$$
B_{x}=B_{0}\left(-1+\tanh \frac{y-y_{B}}{\delta}+\tanh \frac{y_{T}-y}{\delta}\right)
$$

where $B_{0}=0.07$, the positions of the two current sheets are $y_{B}=-3.2$ and $y_{T}=3.2$, respectively, and the width of the currents sheets are controlled by $\delta=0.5$. The electrons have a velocity in the z-direction to generate current equal to the curl of the magnetic field, i.e, $J_{z}=n_{e} q_{e} u_{e, z}=-\partial B_{x} / \partial y$. The ion pressure $p_{i}$ is uniform in the whole domain. Far away from the current sheets, the ion plasma beta is 1 , and the electron pressure is $1 / 5$ of the ion pressure. Near the current sheet, the electrons are heated to balance the magnetic field gradient force, which is the same as the Lorentz force $-n_{e} q_{e} u_{e, z} B_{x}$. This unperturbed initial condition is in fluid force balance [30].

A perturbation is added to excite the reconnection [31]. The magnetic field perturbation vector potential is $A_{x}=0, A_{y}=0$ and:

$$
\begin{aligned}
A_{z}=A_{0} B_{0}\{ & -e^{-\frac{\left(x-x_{T}\right)^{2}}{G_{x}^{2}}-\frac{\left(y-y_{T}\right)^{2}}{G_{y}^{2}}} \cos \left[k_{x}\left(x-x_{T}\right)\right] \cos \left[k_{y}\left(y-y_{T}\right)\right] \\
& \left.+e^{-\frac{\left(x-x_{B}\right)^{2}}{G_{x}^{2}}-\frac{\left(y-y_{B}\right)^{2}}{G_{y}^{2}}} \cos \left[k_{x}\left(x-x_{B}\right)\right] \cos \left[k_{y}\left(y-y_{B}\right)\right]\right\}
\end{aligned}
$$

where the perturbation amplitude is set by $A_{0}=0.1$, the locations along the top and bottom current sheets are $x_{T}=6.4$ and $x_{B}=-6.4$, respectively, the width of Gaussian profiles are $G_{x}=G_{y}=0.5$, and the wave vectors are 
$k_{x}=2 \pi / 25.6$ and $k_{y}=2 \pi / 12.8$. Since these two reconnection sites, i.e., the bottom left one at $\left(x_{B}, y_{B}\right)$ and the top right one at $\left(x_{T}, y_{T}\right)$, produce the same signatures, we only plot and discuss the bottom left reconnection site for simplicity.

For the simulations shown in Figures 8, 9 and 10, the grid resolution is $\Delta x=0.05$ and the time step is $\Delta t=0.1$. There are 900 macro-particles per cell per species. The simulation results at $t=400$ are shown. Figure 8 shows the net charge $\rho_{c}$, electric field $E_{x}$ and the error in Gauss's law for iPIC3D and ECSIM. iPIC3D produces good quality results for this test. Near the reconnection site, the divergent field-aligned electric field $E_{x}$ is well resolved, a double-sandwich structure of the net charge in the center of the reconnection site is captured, and the error is small and dominated by the random particle noise. However, $\rho_{c}$ and $E_{x}$ of ECSIM are dominated by the unphysical oscillations along the separatrices, and the huge error indicates that Gauss's law is dramatically violated. The ECSIM simulation shown here uses $\theta=0.5$, and the simulation with $\theta=0.51$ does not alleviate the issue. To the best of our knowledge, the double-sandwich net charge structure has not been discussed in detail before, but some published high-resolution PIC simulations have provided the evidence of the existence of this structure. For example, the static electric field $E_{z}$, which is $E_{y}$ in the current paper, shown in Figure 1(a) of [32] and Figure 3(b) of [33] is corresponding to the double-sandwich net charge.

Comparing the GL-ECSIM-1 and GL-ECSIM-2 results in Figure 9 demonstrates that the extended stencil discretization of $\nabla \cdot \mathbf{E}$ helps to reduce the noise. All the position correction methods produce essentially the same net charge structure (GL-ECSIM-2 to GL-ECSIM-5). The error in Gauss's law is about 5 orders smaller than the net charge density in the simulations employing the accurate correction method (GL-ECSIM-1 to GL-ECSIM-3), and it is about 1 order smaller for the approximate corrections (GL-ECSIM- 4 and GL-ECSIM-5). When the accurate correction is only applied to electrons (GL-ECSIM-1 and GL-ECSIM-2), the typical maximum particle displacement is $4.5 \%, 0.12 \%$ and $0.002 \%$ of the cell size for the three linearized corrections. These values reduce by a factor of 2 when both electrons and ions are corrected (GL-ECSIM-3). For the approximate corrections GL-ECSIM-4 and GL-ECSIM-5, the typical maximum displacement is about $3 \%$ of the cell size.

Figure 10 shows the total energy variation. For ECSIM with $\theta=0.5$ (ECSIM-1), the energy is conserved, the small error corresponds to the ac- 
curacy of the iterative implicit electric field solver. ECSIM with $\theta=0.51$ (ECSIM-2) dissipates $0.5 \%$ of the total energy after 4000 iterations. The plots of GL-ECSIM-1 and ECSIM-2 are overlapped with each other because the particle position correction does not change the energy. The extended stencil discretization of $\nabla \cdot \mathbf{E}$ (GL-ECSIM-2) dissipates $3 \%$ of the energy, which is still a relatively small value. The energy variation for other correction methods (GL-ECSIM-3 to GL-ECSIM-5) are essentially the same as GL-ECSIM-2. As a comparison, the total energy of the iPIC3D simulation reduces about $3.5 \%$

The normalized wall time for each simulation is presented in Table 1 . From the timing results, we conclude:

- In our implementation, ECSIM is about twice slower than iPIC3D.

- For the accurate correction method (GL-ECSIM-1 to GL-ECSIM-3), the correction takes $30 \%$ to $40 \%$ of the total simulation time.

- Correcting all species (GL-ECSIM-3) is about 10\% slower than correcting one species only (GL-ECSIM-1 and GL-ECSIM-2).

- The approximate correction methods only take about $10 \%$ or less of the total wall time.

In practice, we prefer the approximate global correction method since it reaches a balance between robustness and efficiency. The approximate local correction method is even faster, but it is less robust and accurate for some challenging problems.

Figure 11 shows the results with the approximate global correction for grid resolution $0.2,0.1,0.05$ and 0.025 . The CFL number is fixed and the corresponding time steps are 0.4,0.2, 0.1 and 0.05, respectively. All the simulations capture the Hall magnetic field $B_{z}$, even the electron flows, such as $u_{e, x}$, very well. Once the grid resolution is close to or higher than half of the electron skin depth $d_{e}=0.18$, the details of the off-diagonal electron pressure terms are also well resolved, while the simulation with $\Delta x=0.2$ is too diffusive to capture these details. The pressure components presented here is similar to other high-resolution PIC simulations, such as the Figure 9 in [34. The double-sandwich structure of the net charge is even harder to capture. Even the simulation with $\Delta x=0.1$ does not resolve this structure well. The normalized reconnection rate is shown in Figure 12. The four simulations 
with different grid resolution have the same normalized reconnection rate of 0.07. The algorithm to calculate the reconnection rate can be found in [30]. These four simulations demonstrate that the GL-ECSIM method converges well with increasing grid resolution, and a variety of reconnection related structures can be captured once the grid resolution is close to or higher than half of the electron skin depth.

\subsection{Weibel instability}

Finally, we perform the 1D Weibel instability test to quantitatively prove that the particle correction methods do not interfere with properly capturing the growth and evolution of this instability.

The simulation is performed on a $1 \mathrm{D}$ domain of size $L_{x}=2 \pi d_{e}$, resolved by cells of size $\Delta x=L_{x} / 64$ and time step $\Delta t=0.05 / \omega_{p e} .400$ particles per cell per species are used. Each of the two counter-streaming electron beams has a speed of $0.8 c$ along the positive or negative $y$-direction. The thermal velocity of the electrons is $u_{e, t h}=0.01 c$. The ions are uniformly distributed to satisfy the charge neutrality requirement, but the ions are much colder and heavier than the electrons $\left(m_{i} / m_{e}=10^{4}\right.$ and $\left.u_{i, t h}=10^{-8} \mathrm{c}\right)$, so that the ions do not move essentially. The linear theory [35] predicts the growth rate of the mode with wavelength $\pi d_{e}$ is $\gamma=0.716 \omega_{p e}$. Figure 13 shows that the growth rates are essentially the same for all the simulations, and the rate is close to the analytic value during the linear growth stage.

\section{Conclusion}

In this paper, we introduce the novel GL-ECSIM algorithm, which can satisfy both the total energy conservation and Gauss's law to the accuracy of the iterative solvers. In practice, we need to sacrifice the energy conservation a little bit and introduce a small amount of diffusion to reduce noise and suppress numerical oscillations by using a time centering parameter $\theta=0.51$ instead of 0.5 of the original ECSIM algorithm. In addition, we introduce a linear combination of the original compact stencil (with a 0.9 weight) and a new extended stencil (with 0.1 weight) for the discretization of the $\nabla \cdot \mathbf{E}$ term in the electric field equation. In effect, this adds a dissipation term proportional to the 4th derivative of the electric field, which helps to remove spurious oscillations.

Our 2D reconnection and magnetosphere simulations suggest that the original ECSIM scheme may produce numerical artifacts due to the violation 
of Gauss's law. In order to solve this problem without changing the energy, we design a class of new algorithms to correct the particle positions after each ECSIM update to satisfy Gauss's law. The accurate correction method carefully calculates the displacement of each particle to eliminate the error in Gauss's law accurately while minimizing the norm of the total displacements. This accurate correction method requires a non-linear iterative solver and takes $30 \%$ to $40 \%$ of the total wall time to do the correction. In order to speed up the simulation, we introduce another two approximate methods. The approximate global correction method solves a Poisson's equation to estimate the particle displacement, and the approximate local correction estimates the displacement based on the surrounding errors. The local correction method is faster than the global correction. But the global correction calculate the displacement based on global information, which makes the global correction more robust for challenging problems.

Using the approximate global GL-ECSIM method with its optimal parameter settings, we performed a grid convergence study for the magnetic reconnection problem. We found that the solution converges well with diminishing grid resolution, and it is converged in most variables if the grid resolution is about one half of the electron skin depth.

Our tests demonstrate that the GL-ECSIM is robust and accurate. It has been successfully applied to our ongoing 3D global magnetospheric simulations.

Acknowledgments: This work was supported by the INSPIRE NSF grant PHY-1513379 and the NSF PREEVENTS grant 1663800. Computational resources supporting this work were provided on the Blue Waters super computer by the NSF PRAC grant ACI-1640510, on the Pleiades computer by NASA High-End Computing (HEC) Program through the NASA Advanced Supercomputing (NAS) Division at Ames Research Center, and from Yellowstone (ark:/85065/d7wd3xhc) provided by NCAR's Computational and Information Systems Laboratory, sponsored by the National Science Foundation. 


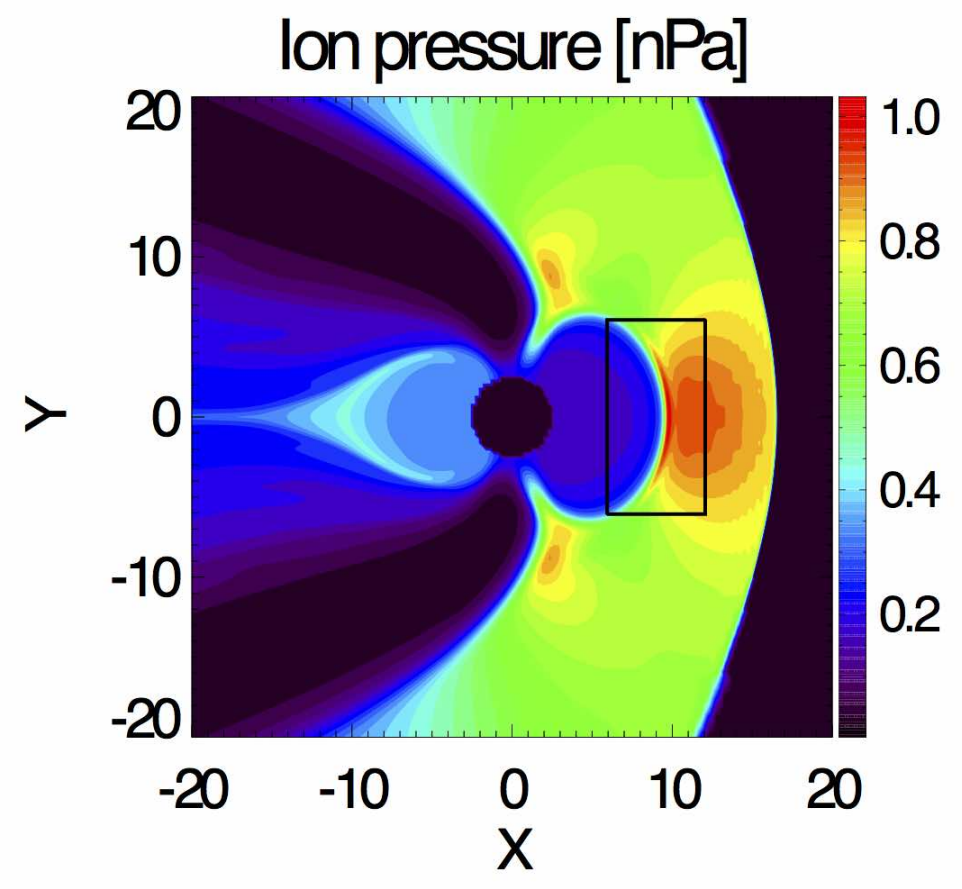

Figure 3: The ion pressure of the 2D magnetosphere simulation. The region inside the black rectangle is simulated by the PIC code. 
(a) IPICDD

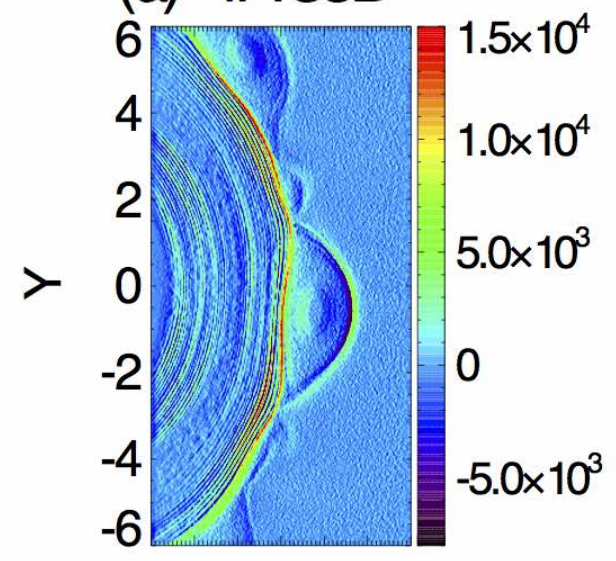

(c) ECSIM-2

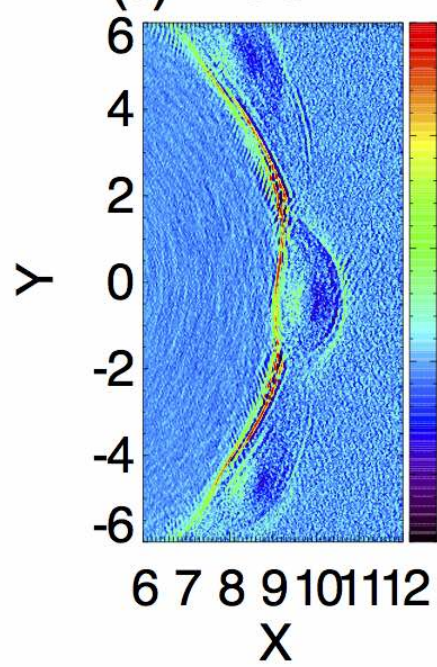

$1.5 \times 10^{4}$

$1.0 \times 10^{4}$

$5.0 \times 10^{3}$

0

$-5.0 \times 10^{3}$ (b) ECSIM-1

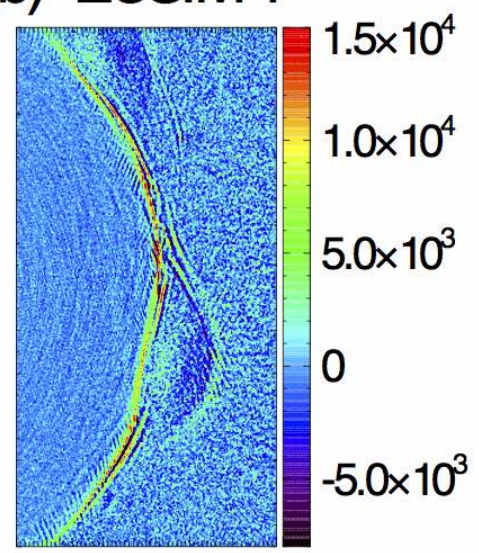

(d) ECSIM-3

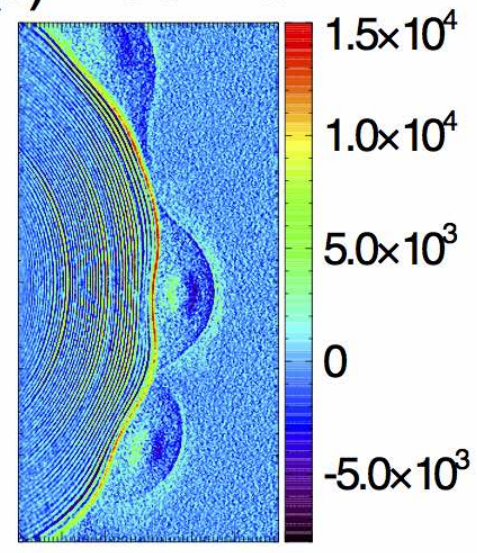

6789101112 $\mathrm{X}$

Figure 4: The electric field $E_{x}[\mathrm{nT} \mathrm{km} / \mathrm{s}]$ of the $2 \mathrm{D}$ magnetosphere simulations inside the PIC domain at $t=400 \mathrm{~s}$ with four different simulation parameters described in Table 1 . iPIC3D produces short-wavelength oscillations inside the magnetosphere. ECSIM with $\theta=0.5$ (ECSIM-1) generates more noise in the magnetosheath than ECSIM with $\theta=0.5$ (ECSIM-2). There are some spurious small scale oscillations near the magnetopause and around the edge of a plasmoid for both ECSIM-1 and ECSIM-2. If the pseudo-current is used to fix the error in Gauss's law (ECSIM-3), it generates oscillations that are similar to the iPIC3D code. 


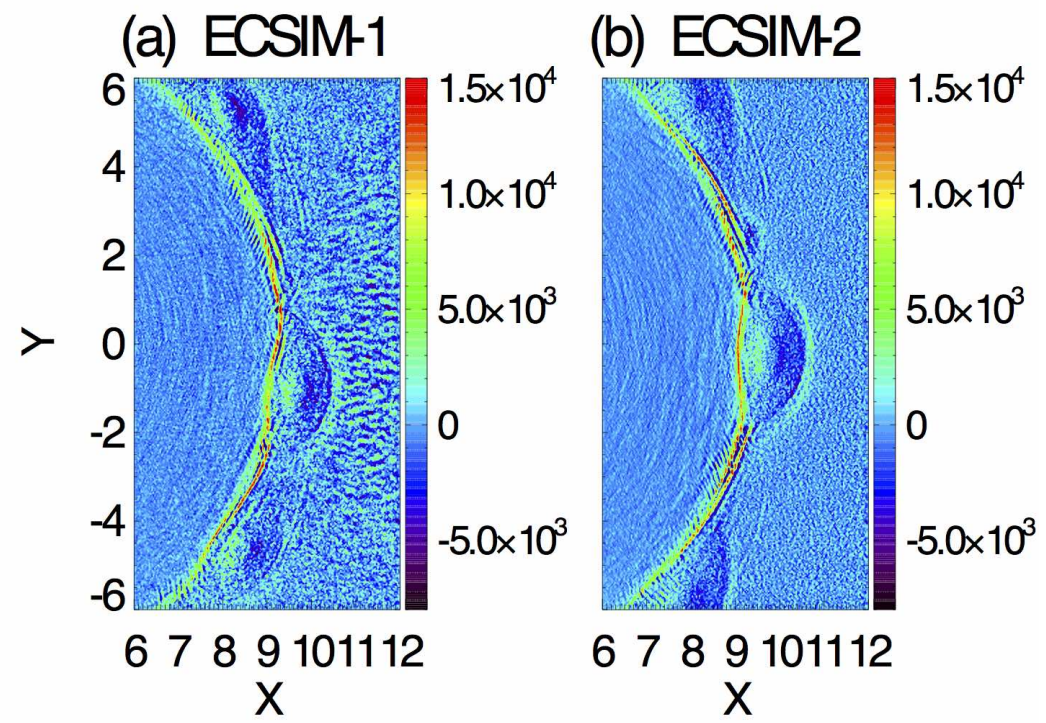

Figure 5: $E_{x}[\mathrm{nT} \mathrm{km} / \mathrm{s}]$ of simulations with $\Delta t=0.1 s$. ECSIM with $\theta=0.5$ (ECSIM-1) produces wave-like structure in the magnetosheath, while ECSIM with $\theta=0.51$ (ECSIM2) does not. 

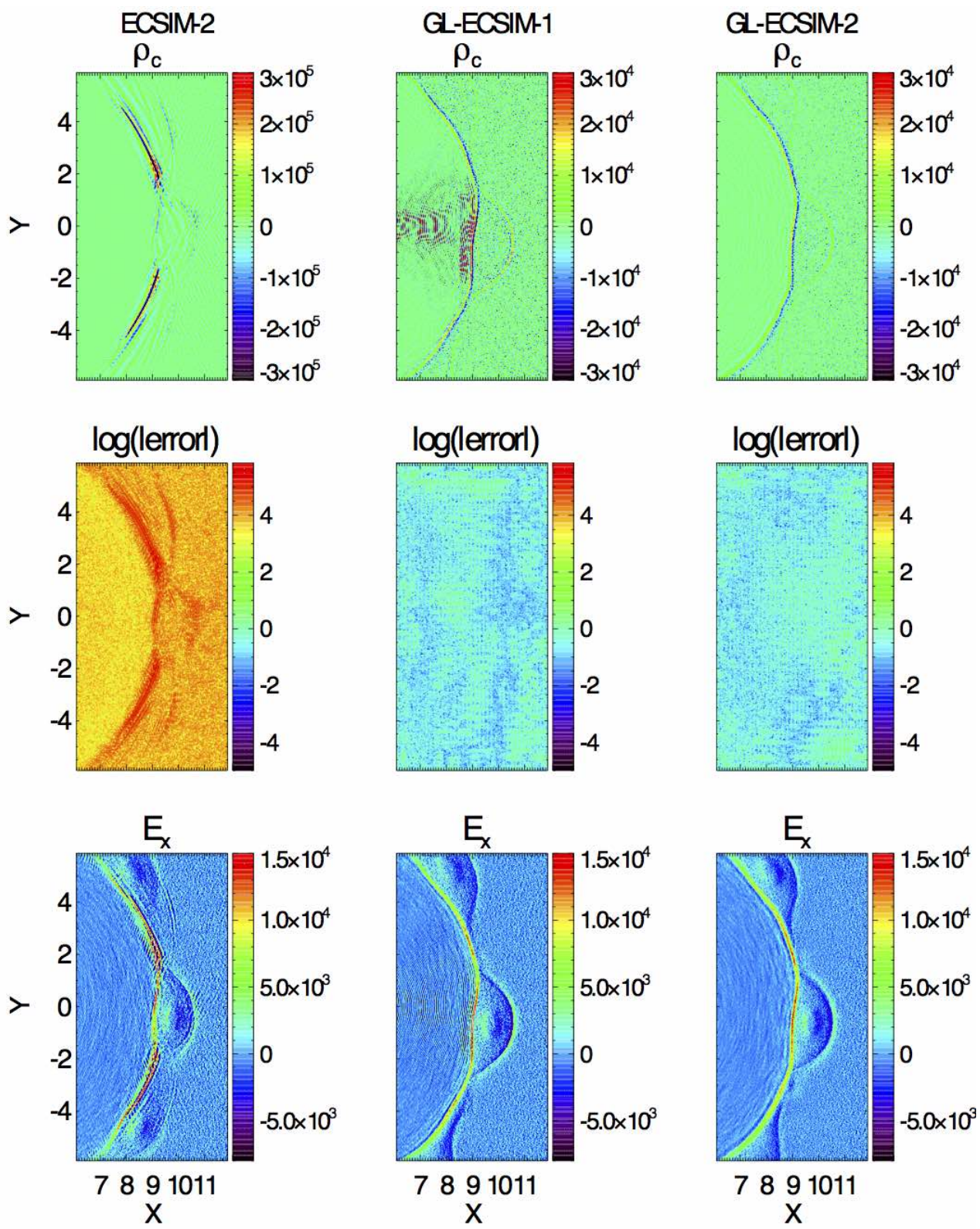

Figure 6: The 2D magnetopause simulations with different parameters. From top to bottom: the net charge $\rho_{c}[\mathrm{nT} / s]$, the absolute value of the error in Gauss's law, defined as $\nabla \cdot \mathbf{E}^{n+1} /(4 \pi)-\rho_{c}^{n+1}$ with units $[\mathrm{nT} / s]$, in logarithmic scale, and the electric field $E_{x}[\mathrm{nT} \cdot \mathrm{km} / \mathrm{s}]$. From left to right: ECSIM with $\theta=0.51$, GL-ECSIM using compact discretization only, and GL-ECSIM with extended stencil for the $\nabla \cdot \mathbf{E}$ discretization. See Table 1 for more details about the parametege. We note that the color bar scale of the net charge density $\rho_{c}$ for ECSIM-2 is different from that of the others. 

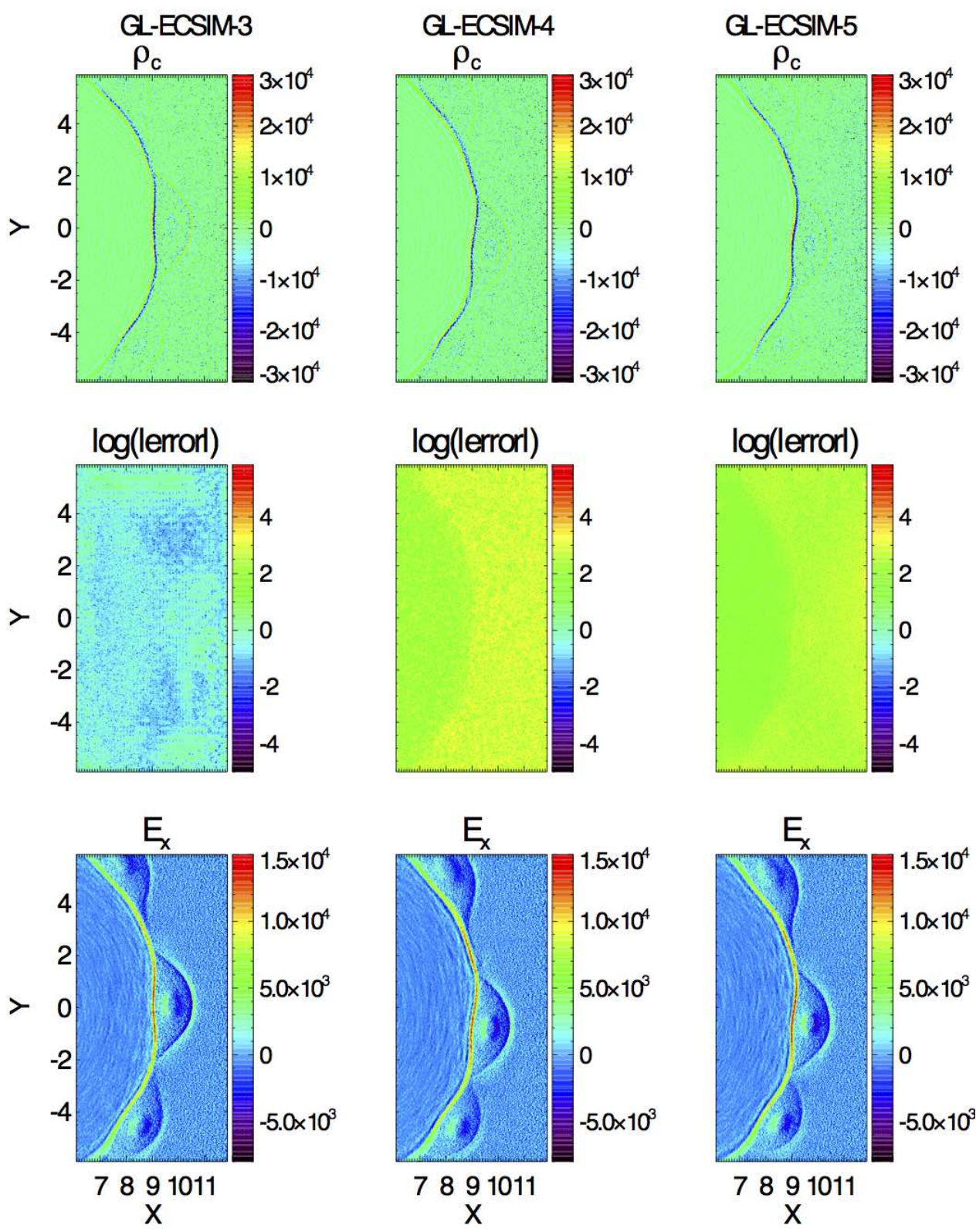

Figure 7: The same variables as in Figure 6. From left to right: the accurate correction for all species, the approximate global correction, and the approximate local correction. 


\section{iPIC3D}
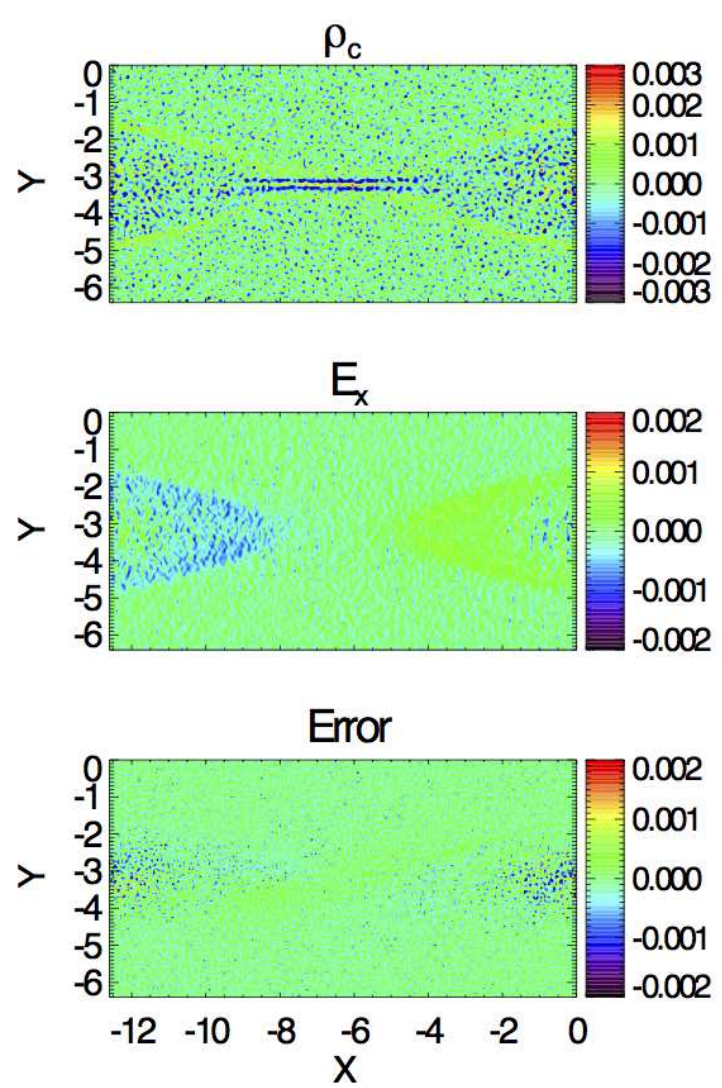

\section{ECSIM-1}
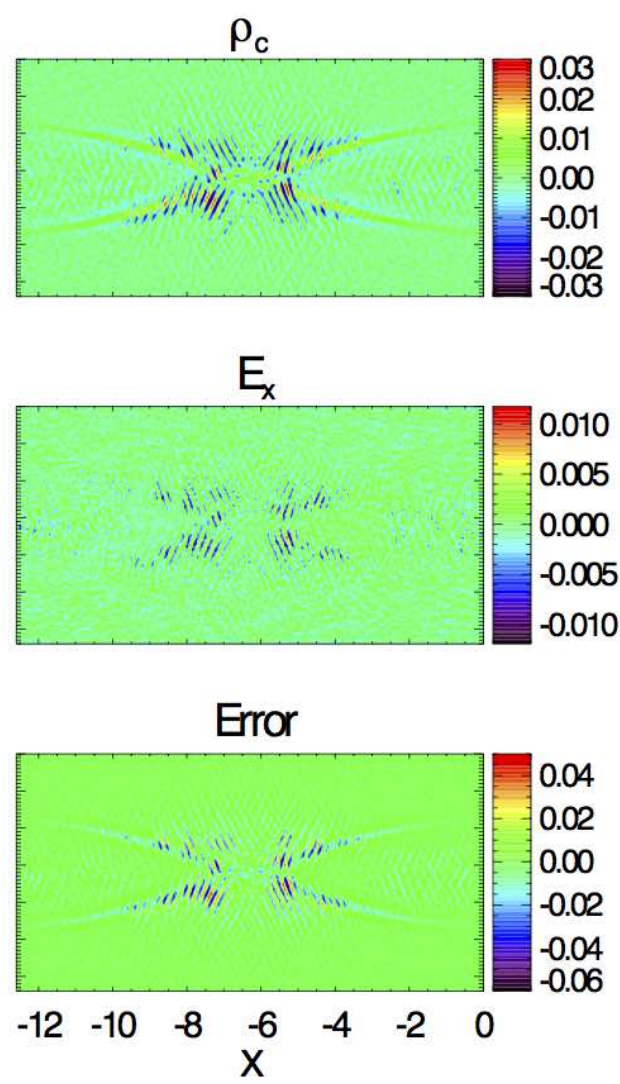

Figure 8: The bottom left reconnection site of the double-current-sheets reconnection simulations at $t=400$. The left panels show the iPIC3D simulation results, and the right panels show the results of ECSIM with $\theta=0.5$. From top to bottom: the net charge $q$, the electric field $E_{x}$ and the error in Gauss's law, defined as $\nabla \cdot \mathbf{E}^{n+1} /(4 \pi)-\rho_{c}^{n+1}$. All these variables are in normalized units. $q$ and $E_{x}$ have the same units. The cell size is $\Delta x=0.05$, and the time step is $\Delta t=0.1$. The results of ECSIM with $\theta=0.51$ are not presented here, but they are very similar to the right panels above. 


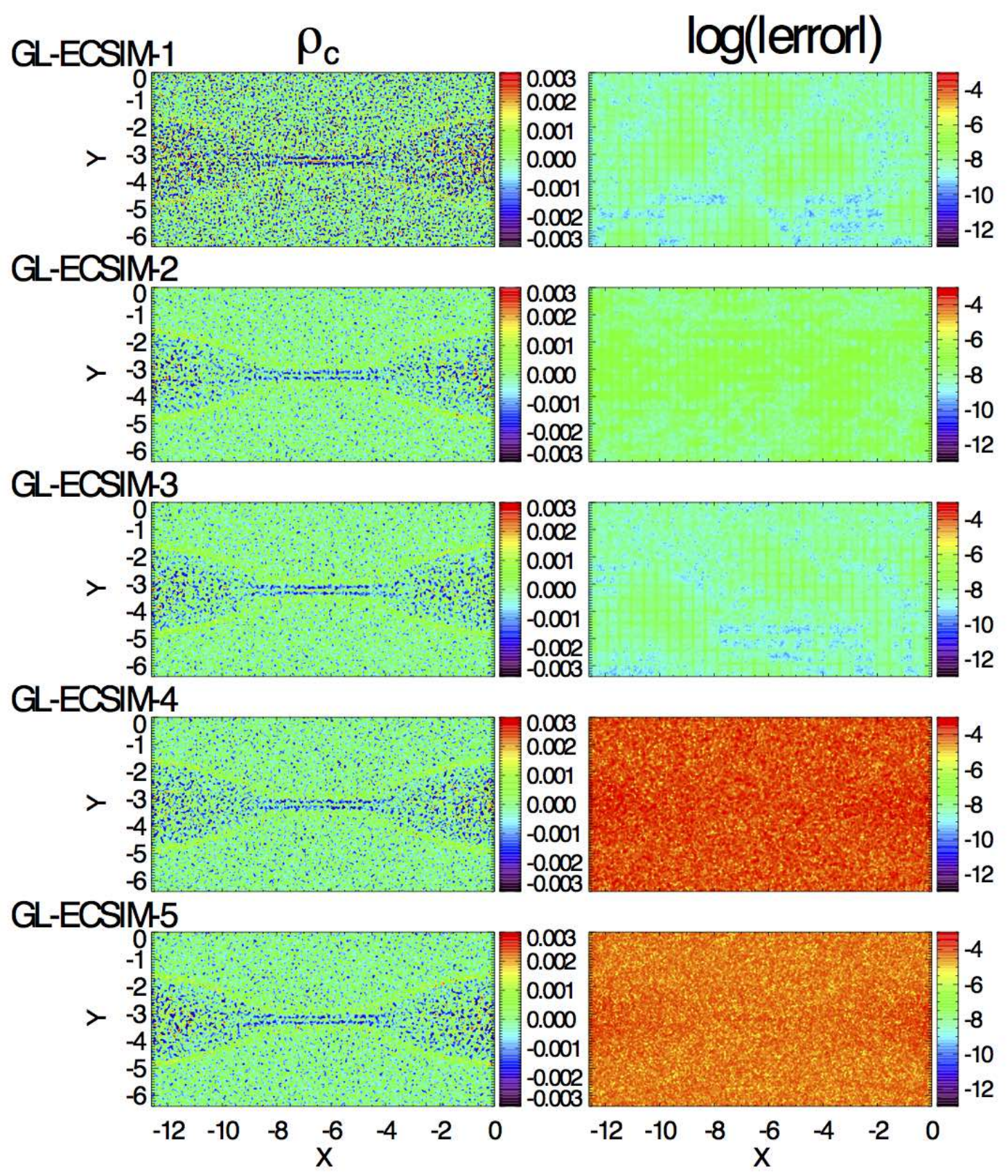

Figure 9: The net charge density $q$ (left pâkel) and the absolute value of the error $\nabla$. $\mathbf{E}^{n+1} /(4 \pi)-\rho_{c}^{n+1}$ in Gauss's law (right panel) in logarithmic scale. The results for different parameters (see Table 1) are presented from top to bottom. 


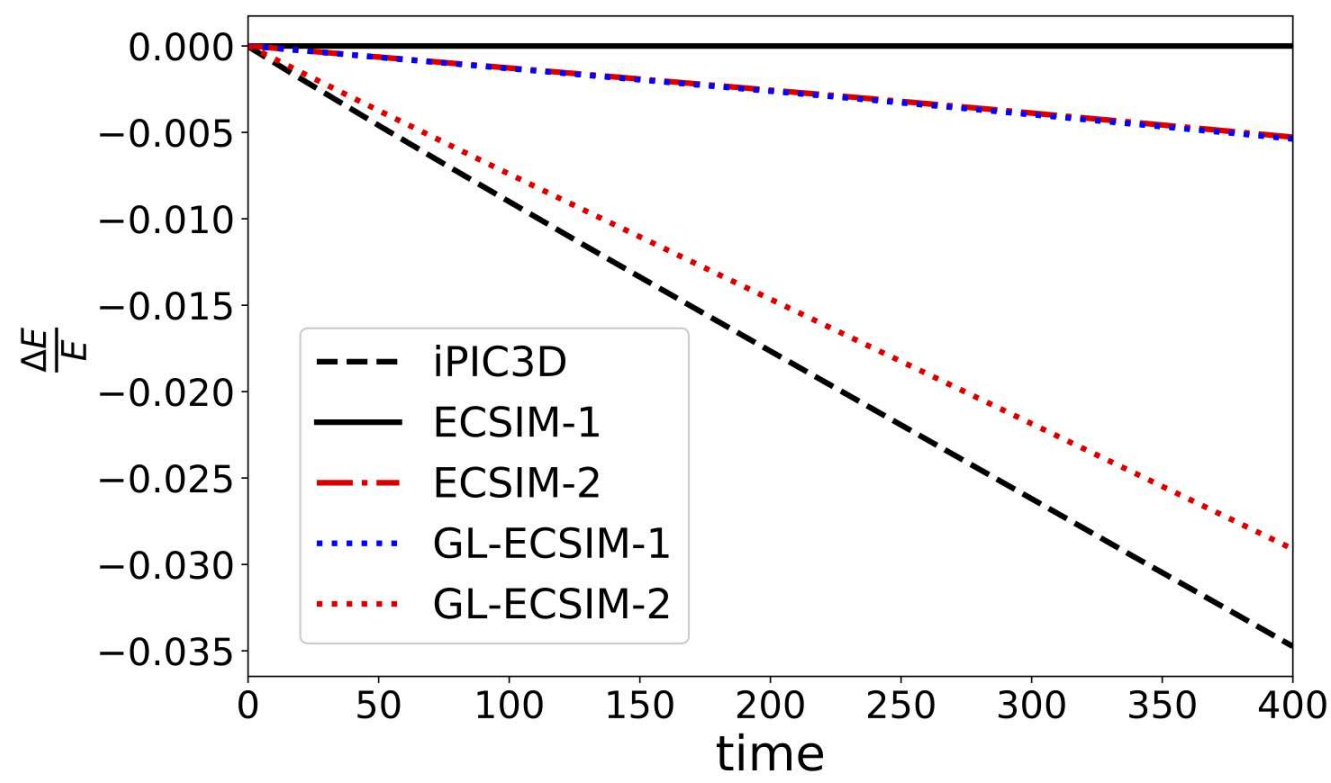

Figure 10: The total energy variation of the double-current-sheet simulations for different schemes (see Table 1). 


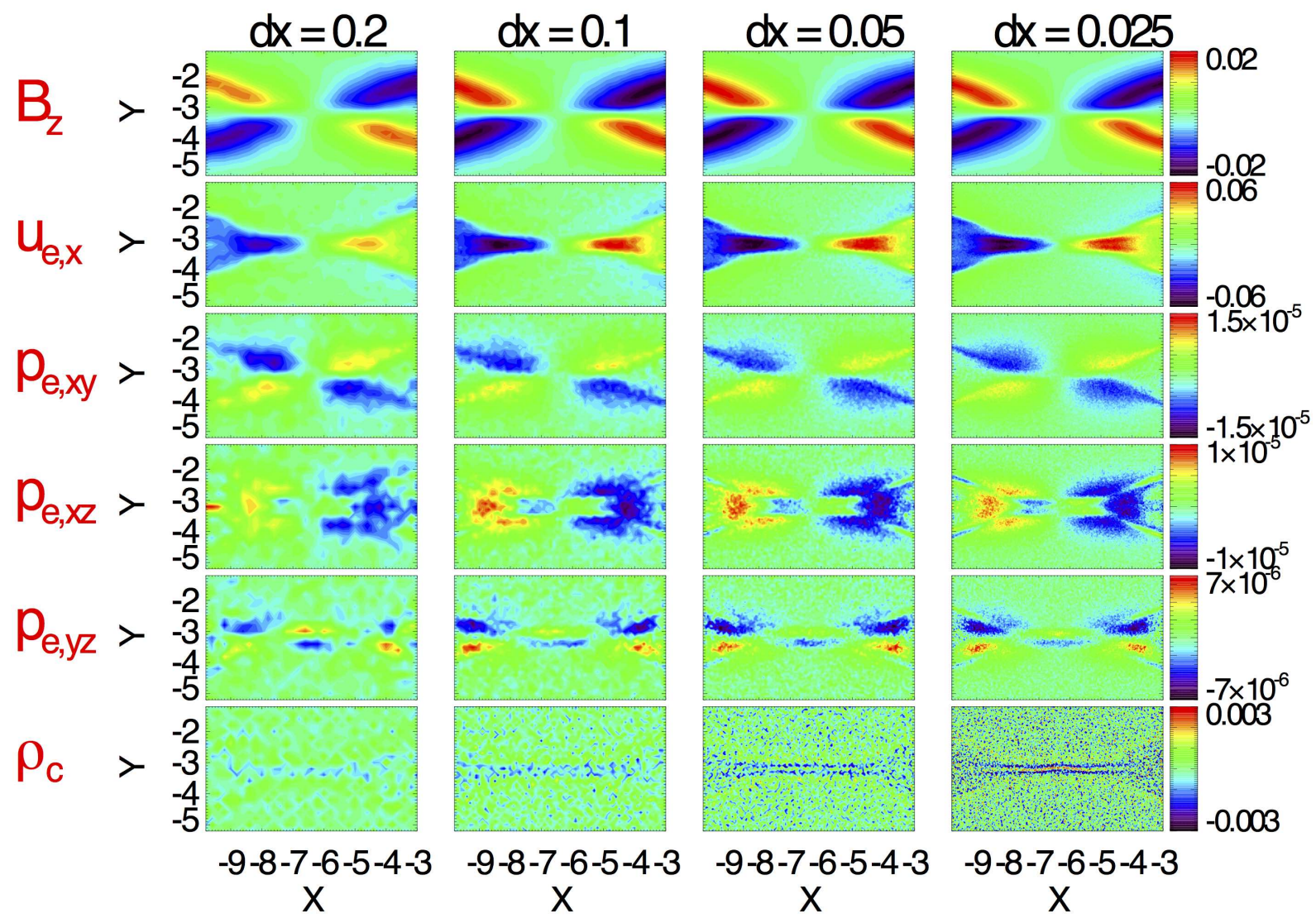

Figure 11: The grid convergence study of the double-current-sheet simulation with the approximate global correction method (GL-ECSIM-4 in Table 1). From top to bottom: the out-of-plane Hall magnetic field $B_{z}$, the electron jet velocity $u_{e, x}$, the three electron off-diagonal pressure tensor elements $p_{e, x y}, p_{e, x z}$ and $p_{e, y z}$, and the net charge density $\rho_{c}$ at $t=400$ are shown in normalized units. From left to right, the cell sizes are $\Delta x=0.2$, $\Delta x=0.1, \Delta x=0.05$ and $\Delta x=0.025$, and the corresponding time steps are $\Delta t=0.4$, $\Delta t=0.2, \Delta t=0.1$ and $\Delta t=0.05$, respectively. 


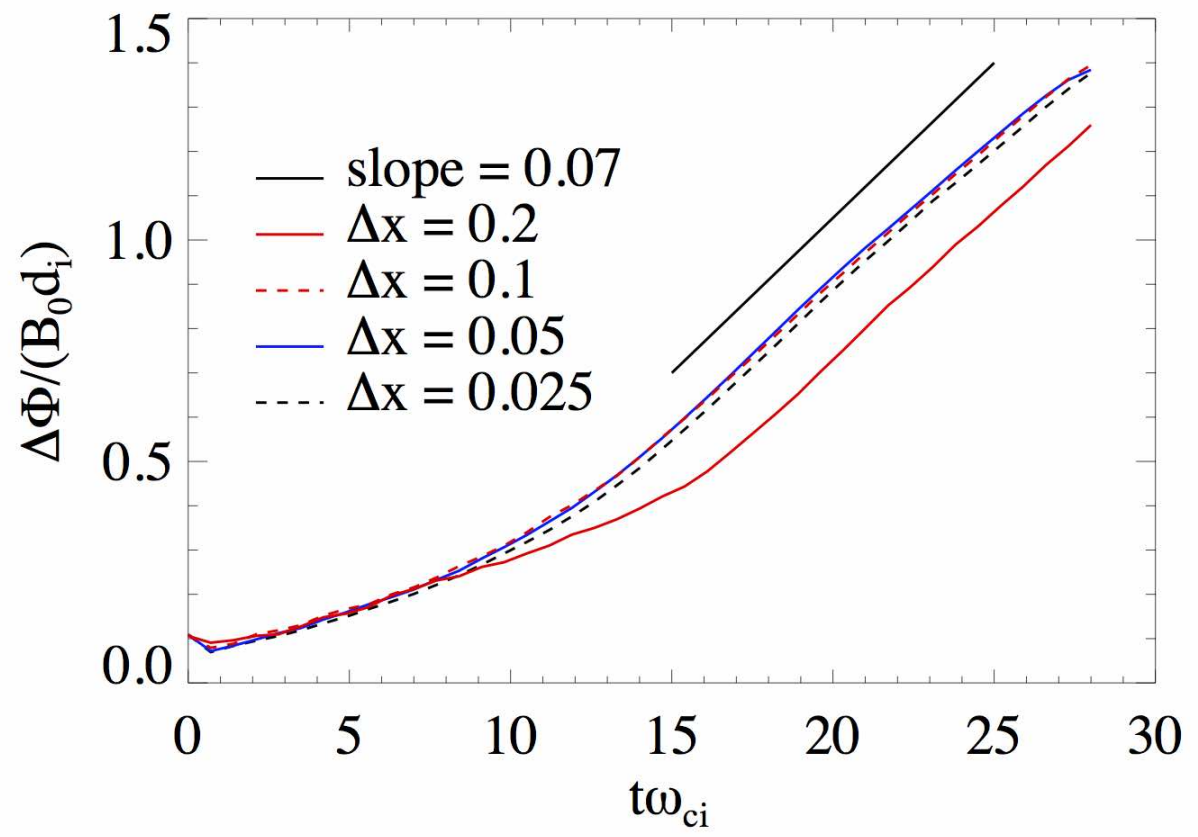

Figure 12: The reconnection rate for the simulations shown in Figure 11. All simulations have a reconnection rate of $\sim 0.07$. 


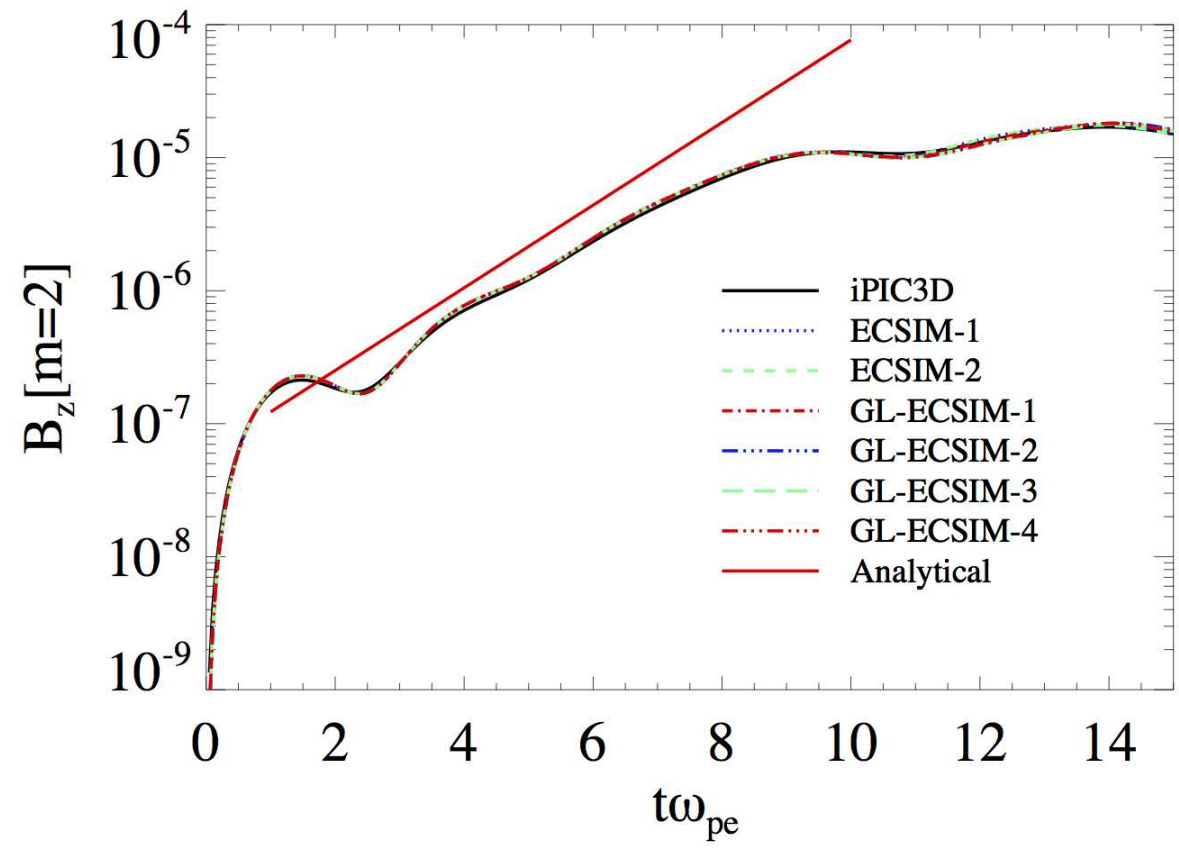

Figure 13: The growth of the Weibel instability. The analytic growth rate is $\gamma=0.716 \omega_{p e}$. The particle correction methods do not change the growth rate at all. 


\section{References}

[1] S. Markidis, G. Lapenta, The energy conserving particle-in-cell method, Journal of Computational Physics 230 (18) (2011) 7037-7052.

[2] G. Chen, L. Chacón, D. C. Barnes, An energy-and charge-conserving, implicit, electrostatic particle-in-cell algorithm, Journal of Computational Physics 230 (18) (2011) 7018-7036.

[3] G. Chen, L. Chacon, A multi-dimensional, energy-and chargeconserving, nonlinearly implicit, electromagnetic vlasov-darwin particle-in-cell algorithm, Computer Physics Communications 197 (2015) 73-87.

[4] L. Chacón, G. Chen, A curvilinear, fully implicit, conservative electromagnetic pic algorithm in multiple dimensions, Journal of Computational Physics 316 (2016) 578-597.

[5] G. Lapenta, Exactly energy conserving semi-implicit particle in cell formulation, Journal of Computational Physics 334 (2017) 349-366.

[6] J. P. Boris, Relativistic plasma simulation-optimization of a hybrid code (1970) 3-67.

[7] C. K. Birdsall, A. B. Langdon, Plasma physics via computer simulation, CRC Press, 2014.

[8] B. Marder, A method for incorporating Gauss' law into electromagnetic PIC codes, J. Comput. Phys. 68 (1987) 48-55.

[9] A. B. Langdon, On enforcing gauss' law in electromagnetic particle-incell codes, Computer Physics Communications 70 (3) (1992) 447-450.

[10] F. Assous, P. Degond, E. Heintze, P.-A. Raviart, J. Segré, On a finiteelement method for solving the three-dimensional maxwell equations, Journal of Computational Physics 109 (2) (1993) 222-237.

[11] C.-D. Munz, P. Omnes, R. Schneider, E. Sonnendrücker, U. Voss, Divergence correction techniques for maxwell solvers based on a hyperbolic model, Journal of Computational Physics 161 (2) (2000) 484-511.

[12] O. Birdsall, Relativistic plasmas, Benjamin, New York, 1968. 
[13] R. Morse, C. Nielson, Numerical simulation of the weibel instability in one and two dimensions, The Physics of Fluids 14 (4) (1971) 830-840.

[14] J. Villasenor, O. Buneman, Rigorous charge conservation for local electromagnetic field solvers, Computer Physics Communications 69 (2-3) (1992) 306-316.

[15] T. Z. Esirkepov, Exact charge conservation scheme for particle-in-cell simulation with an arbitrary form-factor, Computer Physics Communications 135 (2) (2001) 144-153.

[16] T. Umeda, Y. Omura, T. Tominaga, H. Matsumoto, A new charge conservation method in electromagnetic particle-in-cell simulations, Computer Physics Communications 156 (1) (2003) 73-85.

[17] I. V. Sokolov, Alternating-order interpolation in a charge-conserving scheme for particle-in-cell simulations, Comp. Phys. Comm. 184 (2013) 320. doi:10.1016/j.cpc.2012.09.015.

[18] J. W. Eastwood, The virtual particle electromagnetic particle-mesh method, Computer Physics Communications 64 (2) (1991) 252-266.

[19] J. Eastwood, W. Arter, N. Brealey, R. Hockney, Body-fitted electromagnetic pic software for use on parallel computers, Computer Physics Communications 87 (1-2) (1995) 155-178.

[20] S. Markidis, G. Lapenta, Rizwan-Uddin, Multi-scale simulations of plasma with ipic3d, Mathematics and Computers in Simulation 80 (2010) 1509-1519. doi:10.1016/j.matcom.2009.08.038.

[21] L. K. S. Daldorff, G. Tóth, T. I. Gombosi, G. Lapenta, J. Amaya, S. Markidis, J. U. Brackbill, Two-way coupling of a global Hall magnetohydrodynamics model with a local implicit Particle-in-Cell model, J. Comput. Phys. 268 (2014) 236. doi:10.1016/j.jcp.2014.03.009.

[22] G. Tóth, X. Jia, S. Markidis, B. Peng, Y. Chen, L. Daldorff, V. Tenishev, D. Borovikov, J. Haiducek, T. Gombosi, A. Glocer, J. Dorelli, Extended magnetohydrodynamics with embedded particle-in-cell simulation of ganymede's magnetosphere, J. Geophys. Res. 121. doi: 10.1002/2015JA021997. 
[23] Y. Chen, G. Tóth, P. Cassak, X. Jia, T. I. Gombosi, J. A. Slavin, S. Markidis, I. B. Peng, V. K. Jordanova, M. G. Henderson, Global three-dimensional simulation of earth's dayside reconnection using a two-way coupled magnetohydrodynamics with embedded particlein-cell model: Initial results, Journal of Geophysical Research: Space Physics 122 (10) 10,318-10,335. arXiv:https://agupubs. onlinelibrary.wiley.com/doi/pdf/10.1002/2017JA024186, doi:10.1002/2017JA024186. URL https://agupubs.onlinelibrary.wiley.com/doi/abs/10. 1002/2017JA024186

[24] G. Tóth, Y. Chen, T. I. Gombosi, P. Cassak, S. Markidis, I. B. Peng, Scaling the ion inertial length and its implications for modeling reconnection in global simulations, Journal of Geophysical Research: Space Physics 122 (10). doi:10.1002/2017JA024189.

URL https://agupubs.onlinelibrary.wiley.com/doi/abs/10. 1002/2017JA024189

[25] D. Gonzalez-Herrero, E. Boella, G. Lapenta, Performance analysis and implementation details of the energy conserving semi-implicit method code (ecsim), Computer Physics Communications.

[26] L. Chacón, D. A. Knoll, A 2d high- $\beta$ hall mhd implicit nonlinear solver, Journal of Computational Physics 188 (2) (2003) 573-592.

[27] M. Tanaka, Macroscale implicit electromagnetic particle simulation of magnetized plasmas, Journal of Computational Physics 79 (1) (1988) 209-226.

[28] M. Tanaka, The macro-em particle simulation method and a study of collisionless magnetic reconnection, Computer physics communications 87 (1-2) (1995) 117-138.

[29] J. Birn, J. F. Drake, M. A. Shay, B. N. Rogers, R. E. Denton, M. Hesse, M. Kuznetsova, Z. W. Ma, A. Bhattacharjee, A. Otto, P. L. Pritchett, Geospace Environmental Modeling (GEM) magnetic reconnection challenge, J. Geophys. Res. 106 (A3) (2001) 3715-3720. doi:10.1029/1999JA900449. 
[30] Z. Huang, G. Tóth, B. van der Holst, Y. Chen, T. I. Gombosi, A sixmoment multi-fluid plasma model, Journal of Computational Physics, submitted.

[31] G. Lapenta, D. Gonzalez-Herrero, E. Boella, Multiple-scale kinetic simulations with the energy conserving semi-implicit particle in cell method, Journal of Plasma Physics 83 (2).

[32] R. Guo, Z. Pu, Y. Wei, Current structure and flow pattern on the electron separatrix in reconnection region, Geoscience Letters 4 (1) (2017) 18.

[33] J. Ng, J. Egedal, A. Le, W. Daughton, Phase space structure of the electron diffusion region in reconnection with weak guide fields, Physics of Plasmas 19 (11) (2012) 112108.

[34] L. Wang, A. H. Hakim, A. Bhattacharjee, K. Germaschewski, Comparison of multi-fluid moment models with particle-in-cell simulations of collisionless magnetic reconnection, Physics of Plasmas 22 (1) (2015) 012108 .

[35] E. S. Weibel, Spontaneously growing transverse waves in a plasma due to an anisotropic velocity distribution, Physical Review Letters 2 (3) (1959) 83. 